INSTITUT NATIONAL DE RECHERCHE EN INFORMATIQUE ET EN AUTOMATIQUE

\title{
A convex approach to robust stability for linear systems with uncertain scalar parameters
}

\author{
Pierre-Alexandre Bliman
}

\section{$\mathbf{N}^{\circ} 4316$}

Novembre 2001

THÈME 4 



\title{
A convex approach to robust stability for linear systems with uncertain scalar parameters
}

\author{
Pierre-Alexandre Bliman* \\ Thème 4 - Simulation et optimisation \\ de systèmes complexes \\ Projet Sosso
}

Rapport de recherche $n^{\circ} 4316$ - Novembre $2001-24$ pages

\begin{abstract}
In this report, robust stability for linear systems with several uncertain (complex and/or real) scalar parameters, is studied. Necessary and sufficient conditions for robust stability are given, in terms of solvability of some simple linear matrix inequalities (LMIs). This result constitutes an extension of the characterization by solvability of Lyapunov inequality, of the asymptotic stability for usual linear systems. The related issue of delay-independent stability for linear systems with multiple (noncommensurate) delays is also treated, and it is shown how the approach used here is linked to the search for quadratic LyapunovKrasovskii functional of a special type.

Key-words: Robust stability, real and complex parametric uncertainty, parameter-dependent Lyapunov functions, linear matrix inequalities, $\mu$-analysis, structured singular values, delay systems, delay-independent stability, Kalman-Yakubovich-Popov lemma.
\end{abstract}

* Email : pierre-alexandre.bliman@inria.fr. 


\section{Une approche convexe de la stabilité robuste des systèmes linéaires avec paramètres scalaires incertains}

Résumé : Dans ce rapport est étudiée la stabilité robuste des systèmes linéaires avec plusieurs paramètres scalaires incertains (complexes ou réels). Des conditions nécessaires et suffisantes de stabilité robuste sont données, en termes de faisabilité d'inégalités linéaires matricielles simples. Ce résultat constitue une extension de la caractérisation par la faisabilité de l'inégalité de Lyapunov, de la stabilité asymptotique des systèmes linéaires usuels. Le sujet connexe de la stabilité indépendante des retards pour les systèmes linéaires avec plusieurs retards (non commensurables) est aussi traité, et on montre que l'approche utilisée ici est liée à la recherche de fonctionnelles quadratiques de Lyapunov-Krasovskii d'un type spécial.

Mots-clés : Stabilité robuste, incertitude paramétrique réelle et complexe, fonctions de Lyapunov dépendant de paramètres, inégalités linéaires matricielles, $\mu$-analyse, valeurs singulières structurées, systèmes à retards, stabilité indépendante du retard, lemme de Kalman-Yakubovich-Popov. 


\section{Introduction}

We study in this paper the robust asymptotic stability of finite-dimensional linear systems subject to several scalar parametric uncertainties, namely:

$$
\dot{x}=\left(A_{0}+z_{1} A_{1}+\cdots+z_{m} A_{m}\right) x,
$$

where $A_{0}, A_{1}, \ldots, A_{m} \in \mathbb{C}^{n \times n}$. Here, the uncertain scalar parameters $z_{i}$ may be complex or real numbers. In the latter case, for sake of clarity, we shall rather write $r_{i}$.

It is a well-known fact that asymptotic stability of system (1) without uncertainty $\left(z_{1}=\cdots=z_{m}=0\right)$ is equivalent to existence of a hermitian matrix $P \in \mathbb{C}^{n \times n}$ such that

$$
P>0, A_{0}^{*} P+P A_{0}<0 \text {. }
$$

This is the well-known Lyapunov inequality. This approach is related to the search for a Lyapunov function of the form $x(t)^{*} P x(t)$, definite positive and decreasing along the trajectories of (1).

This approach has been extended in different ways, in order to consider uncertain systems (1). In the various existing approaches, one usually considers a set of constant systems, typically compact and convex: the task is to establish whether all the systems in this set are asymptotically stable or not. Various types of parameter sets are in consequence associated to (1), usually elliptic or polytopic. In the present paper, we consider the case of constant, noncorrelated, parameters, with values in closed unit balls of $\mathbb{R}$ or $\mathbb{C}$. In other words, we wish to find a numerically tractable test of the existence of a hermitian matrix $P\left(z_{1}, \ldots, z_{m}\right)$ such that

$P\left(z_{1}, \ldots, z_{m}\right)>0,\left(A_{0}+z_{1} A_{1}+\cdots+z_{m} A_{m}\right)^{*} P\left(z_{1}, \ldots, z_{m}\right)+P\left(z_{1}, \ldots, z_{m}\right)\left(A_{0}+z_{1} A_{1}+\cdots+z_{m} A_{m}\right)<0$,

for any $\left|z_{i}\right| \leq 1, z_{i} \in \mathbb{R}$ or $\mathbb{C}, i=1, \ldots, m$. This problem appears as a parameter-dependent or parametrized $L M I[1]$.

A first way to cope with uncertainty consists in looking for simultaneous Lyapunov function, i.e. for a constant hermitian definite positive $P$ such that $x(t)^{*} P x(t)$ decreases along the trajectories of $(1)$, for any value of $z_{1}, \ldots, z_{m}$ in the convenient product of balls. For bibliography on this notion, quadratic stability, the reader is refered to [6, 72-73]. For convex compact sets of parameters, it is sufficient to verify decreasingness of the candidate Lyapunov function for the values on the boundary. When the parameter set is polytopic, it is enough to check the vertices of the polytope.

The latter approach leads to sufficient conditions for robust stability of (1), that is for asymptotic stability for any value of the $z_{i}$ in the adequate set, which however are far from being necessary. On the other hand, the benefit of the method is to reduce to testing the solvability of LMI problems. The resolution of this latter class of problems is a standard convex optimization problem [6], achievable in polynomial-time, for which very efficient interior-point methods have been developed and are available as toolboxes in widely-spread control-oriented scientific softwares, such as MatlaB or ScIlaB.

Subsequent developments have led to consider parameter-dependent Lyapunov functions [20, 21]. Sufficient conditions for existence of affine parameter-dependent functions $P\left(z_{1}, \ldots, z_{m}\right)$ in $(2)$, are provided in $[15,13,10,11,32]$, and in $[40,41]$ for functions quadratic in the parameters. These approaches too provide numerically checkable LMI conditions, but, although more precise, they still do not constitute necessary conditions for robust stability, and furnish conservative indications. Methods involving piecewise quadratic Lyapunov functions $[42,34]$ and LMIs with augmented number of variables $[16,31]$ may also be found.

In contrast, the results we are about to present here, provide necessary and sufficient conditions for robust stability of system (1). The general principle for their obtention may be explained as follows.

- First, notice that the solution $P\left(z_{1}, \ldots, z_{m}\right)$ of Lyapunov identity

$$
\left(A_{0}+z_{1} A_{1}+\cdots+z_{m} A_{m}\right)^{*} P\left(z_{1}, \ldots, z_{m}\right)+P\left(z_{1}, \ldots, z_{m}\right)\left(A_{0}+z_{1} A_{1}+\cdots+z_{m} A_{m}\right)=-I_{n}
$$

$\mathrm{RR} \mathrm{n}^{\circ} 4316$ 
is analytic wrt the parameters $z_{i}$ and their conjugate $z_{i}^{*}$. Indeed, this fact may be checked from the explicit form

$$
P\left(z_{1}, \ldots, z_{m}\right)=\int_{0}^{+\infty} e^{\left(A_{0}+z_{1} A_{1}+\cdots+z_{m} A_{m}\right)^{*} t} e^{\left(A_{0}+z_{1} A_{1}+\cdots+z_{m} A_{m}\right) t} d t .
$$

As a matter of fact, this suggests that for robustly stable systems, there always exists parameterdependent Lyapunov function $x(t)^{*} P\left(z_{1}, \ldots, z_{m}\right) x(t)$ with $P$ fulfilling $(2)$ and polynomial wrt $z_{i}, z_{i}^{*}$. Basically, this is due to the fact that inequality (2) has to be fulfilled for a compact set of parameters. One hence takes as new unknowns of the problem a positive integer $k$, such that $k-1$ represents the maximal power in the variables $z_{i}, z_{i}^{*}$ of the polynomial $P\left(z_{1}, \ldots, z_{m}\right)$, plus the $k^{m}$ coefficients themselves, which are hermitian matrices of size $n \times n$.

- Second, the conditions that must be verified by the previous coefficients (including the global condition of positivity of $P\left(z_{1}, \ldots, z_{m}\right)$ for all $\left.z_{1}, \ldots, z_{m}\right)$ are transformed into a set of linear matrix inequalities in a total of $m+1$ unknown hermitian matrices. The main tool for this operation is the application, repeated $m$ times, of discrete-time Kalman-Yakubovich-Popov lemma.

This two-step procedure motivates the form of the results presented in the core of the paper, which we now summarize. A family of LMIs is exhibited, indexed by the positive integer $k$ (roughly speaking, the degree in the $z_{i}, z_{i}^{*}$ of a solution of (2)), and whose solvability implies robust stability of system (2). Also, it is shown that solvability for rank $k$ implies solvability for $k^{\prime} \geq k$, so these sufficient conditions are more and more precise (less and less conservative), as the "degree" of the polynomial solution increases. A key issue is that these conditions are also necessary, in the sense that: if robust stability holds, then the corresponding LMIs are fulfilled from a certain rank $k$ and beyond. Thus, the conservatism vanishes asymptotically. Robust stability of system (1) is hence characterized by solvability of LMI problems. Up to our knowledge, no already published method permits, as the present one, to expect total reduction of the approximation error for the class of problems studied here, while avoiding time-consuming frequency sweep.

The paper is organized as follows. In Section 2 are given some notations necessary to the statement of the results. In Section 3 is stated the result corresponding to $m$ complex parameters (Theorem 1 ). The link with the notion of delay-independent stability for linear systems with $m$ discrete delays is clarified in Section 4, where a general class of quadratic Lyapunov-Krasovskii functionals is shown to be naturally associated to the LMIs in Theorem 1. The result corresponding to $m$ real parameters is given in Section 5 (Theorem 3 ). The mixed case may be written down easily, and is not extensively developed here. Complexity issues are examined in Section 6, and the relationship with $\mu$-analysis and real and complex structured singular values is underlined. Numerical example is presented in Section 7, achieved with the LMI solver of the free software ScilaB. Complete proof of Theorems 1 and 3 is given in Section 8. Last, concluding remarks are given in Section 9.

\section{Notations}

The matrices $I_{n}, 0_{n}, 0_{n \times p}$ are resp. the $n \times n$ identity matrix and the $n \times n$ and $n \times p$ zero matrices. The symbol $\otimes$ denotes Kronecker product. The spectrum of a square matrix $M$ is written $\sigma(M)$, and applying the operation $\operatorname{Re}$ to this set, one denotes by $\operatorname{Re} \sigma(M)$ the set $\{\operatorname{Re} s: s \in \sigma(M)\}: \operatorname{Re} \sigma(M)<0$ thus means

that $M$ is Hurwitz. The conjugate and transconjugate of $M$, are denoted $M^{T}$ and $M^{*}$. $\mathbb{N}$ is the set of positive integers. By $\overline{\mathbb{D}}$ is denoted the closed unit ball in $\mathbb{C}$. The unit circle is denoted as the boundary $\partial \mathbb{D}$. By $\overline{\mathbb{C}^{+}}$is meant the closed set of complex numbers with nonnegative real part. Also, for a delay $h \geq 0, x_{t}$ designates the function $x(t+\cdot)$, defined on $[-h, 0]$.

Let $\hat{J}_{k}, \check{J}_{k} \in \mathbb{R}^{k \times(k+1)}$ be defined by

$$
\hat{J}_{k} \stackrel{\text { def }}{=}\left(I_{k} \quad 0_{k \times 1}\right), \check{J}_{k} \stackrel{\text { def }}{=}\left(\begin{array}{ll}
0_{k \times 1} & I_{k}
\end{array}\right) .
$$


Define also, for $k, m \in \mathbb{N}$, the matrices $J_{k}^{m}, 0 \leq i \leq m$, and the matrices $\hat{J}_{k, i}^{m}, \check{J}_{k, i}^{m}, 1 \leq i \leq m$, by:

$$
\begin{aligned}
& J_{k}^{0}=1, J_{k}^{m} \stackrel{\text { def }}{=}\left(\begin{array}{c}
\hat{J}_{k} \otimes \cdots \otimes \hat{J}_{k} \otimes \hat{J}_{k} \\
\hat{J}_{k} \otimes \cdots \otimes \hat{J}_{k} \otimes \breve{J}_{k} \\
\hat{J}_{k} \otimes \cdots \otimes \check{J}_{k} \otimes \hat{J}_{k} \\
\vdots \\
\check{J}_{k} \otimes \hat{J}_{k} \otimes \cdots \otimes \hat{J}_{k}
\end{array}\right), \\
& \hat{J}_{k, 1}^{m} \stackrel{\text { def }}{=}\left(\hat{J}_{k} \otimes \cdots \otimes \hat{J}_{k} \otimes \hat{J}_{k}\right), \check{J}_{k, 1}^{m} \stackrel{\text { def }}{=}\left(\hat{J}_{k} \otimes \cdots \otimes \hat{J}_{k} \otimes \check{J}_{k}\right), \\
& \hat{J}_{k, 2}^{m} \stackrel{\text { def }}{=}\left(\hat{J}_{k} \otimes \cdots \otimes \hat{J}_{k} \otimes I_{k+1}\right), \check{J}_{k, 2}^{m} \stackrel{\text { def }}{=}\left(\hat{J}_{k} \otimes \cdots \otimes \check{J}_{k} \otimes I_{k+1}\right), \ldots \text { until } \\
& \hat{J}_{k, m}^{m} \stackrel{\text { def }}{=}\left(\hat{J}_{k} \otimes I_{k+1} \otimes \cdots \otimes I_{k+1}\right), \check{J}_{k, m}^{m} \stackrel{\text { def }}{=}\left(\check{J}_{k} \otimes I_{k+1} \otimes \cdots \otimes I_{k+1}\right) .
\end{aligned}
$$

Each of the previous sequences of Kronecker products involves overall $m$ terms, and the size of the matrices is given by

$$
J_{k}^{m} \in \mathbb{R}^{(m+1) k^{m} \times(k+1)^{m}}, \quad \hat{J}_{k, i}^{m}, \breve{J}_{k, i}^{m} \in \mathbb{R}^{k^{m-i+1}(k+1)^{i-1} \times(k+1)^{m}}, 1 \leq i \leq m .
$$

The matrices $J_{k}^{m}, \hat{J}_{k, i}^{m}, \check{J}_{k, i}^{m}$ may alternatively be constructed by induction on $m$, as follows:

$$
\begin{aligned}
& J_{k}^{0} \stackrel{\text { def }}{=} 1, J_{k}^{m+1} \stackrel{\text { def }}{=}\left(\begin{array}{c}
\check{J}_{k} \otimes J_{k}^{m} \\
\check{J}_{k} \otimes \underbrace{\hat{J}_{k} \otimes \cdots \otimes \hat{J}_{k}}_{m \text { times }}
\end{array}\right), m \geq 0, \\
& \hat{J}_{k, 1}^{1} \stackrel{\text { def }}{=} \hat{J}_{k}, \hat{J}_{k, i}^{m+1} \stackrel{\text { def }}{=} \hat{J}_{k} \otimes \hat{J}_{k, i}^{m}, 1 \leq i \leq m, \hat{J}_{k, m+1}^{m+1} \stackrel{\text { def }}{=} \hat{J}_{k} \otimes I_{(k+1)^{m}}, m \geq 1, \\
& \breve{J}_{k, 1}^{1} \stackrel{\text { def }}{=} \check{J}_{k}, \check{J}_{k, i}^{m+1} \stackrel{\text { def }}{=} \hat{J}_{k} \otimes \check{J}_{k, i}^{m}, 1 \leq i \leq m, \check{J}_{k, m+1}^{m+1} \stackrel{\text { def }}{=} \check{J}_{k} \otimes I_{(k+1)^{m}}, m \geq 1 \text {. }
\end{aligned}
$$

Last, for $z \in \mathbb{C}$, we define $v_{k}(z) \in \mathbb{C}^{k}$ by

$$
v_{k}(z) \stackrel{\text { def }}{=}\left(\begin{array}{c}
1 \\
\vdots \\
z^{k-1}
\end{array}\right) .
$$

\section{Robust stability of systems with complex parameters}

Theorem 1. The matrix $A_{0}+z_{1} A_{1}+\cdots+z_{m} A_{m}$ is Hurwitz for any $z_{1}, \ldots, z_{m} \in \overline{\mathbb{D}}$, if and only if there exist a positive integer $k$ and $(m+1)$ hermitian matrices

$$
P_{k} \in \mathbb{C}^{k^{m} n \times k^{m} n} \text { and } Q_{k, i} \in \mathbb{C}^{k^{m-i+1}(k+1)^{i-1} n \times k^{m-i+1}(k+1)^{i-1} n}, i=1 \ldots m,
$$

such that the following LMI is solvable:

$$
P_{k}>0, R_{k}^{\mathbb{C}}\left(P_{k}, Q_{k, 1}, \ldots, Q_{k, m}\right)<0,
$$

where $R_{k}^{\mathbb{C}} \in \mathbb{C}^{(k+1)^{m} n \times(k+1)^{m} n}$ is defined as

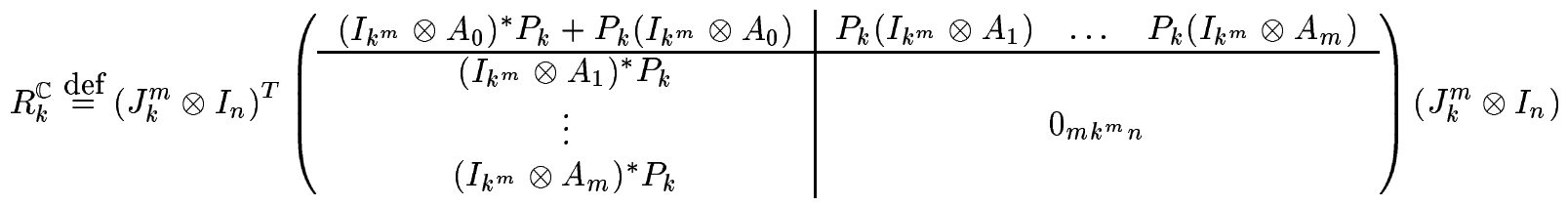

$$
\begin{aligned}
& +\sum_{i=1}^{m}\left(\left(\hat{J}_{k, i}^{m} \otimes I_{n}\right)^{T} Q_{k, i}\left(\hat{J}_{k, i}^{m} \otimes I_{n}\right)-\left(\check{J}_{k, i}^{m} \otimes I_{n}\right)^{T} Q_{k, i}\left(\check{J}_{k, i}^{m} \otimes I_{n}\right)\right) .
\end{aligned}
$$

Moreover,

RR $\mathrm{n}^{\circ} 4316$ 
- if $\mathrm{LMI}_{k}^{\mathbb{C}}$ is solvable, then it is also solvable for indexes $k^{\prime} \geq k$;

- if the matrices $A_{i}, 0 \leq i \leq m$, are real, then the statement holds with real, symmetric, matrices $P_{k}, Q_{k, i}$, $1 \leq i \leq m$.

Proof of Theorem 1 is given in Section 8.2.

In the case $m=0$, problem $\mathrm{LMI}_{k}^{\mathbb{C}}$ simply writes: $\exists P=P^{*}>0, A_{0}^{*} P+P A_{0}<0$. For $m=1$, one gets the following family of LMIs indexed by $k \in \mathbb{N}: \exists P_{k} \in \mathbb{C}^{k n \times k n}, P_{k}=P_{k}^{*}>0, \exists Q_{k} \in \mathbb{C}^{k n \times k n}, Q_{k}=Q_{k}^{*}$,

$$
\begin{aligned}
\left(\begin{array}{c}
\hat{J}_{k} \otimes I_{n} \\
\check{J}_{k} \otimes I_{n}
\end{array}\right)^{T}\left(\begin{array}{cc}
\left(I_{k} \otimes A_{0}\right)^{*} P_{k}+P_{k}\left(I_{k} \otimes A_{0}\right) & P_{k}\left(I_{k} \otimes A_{1}\right) \\
\left(I_{k} \otimes A_{1}\right)^{*} P_{k} & 0_{k n}
\end{array}\right)\left(\begin{array}{l}
\hat{J}_{k} \otimes I_{n} \\
\check{J}_{k} \otimes I_{n}
\end{array}\right) \\
+\left(\hat{J}_{k} \otimes I_{n}\right)^{T} Q_{k}\left(\hat{J}_{k} \otimes I_{n}\right)-\left(\check{J}_{k} \otimes I_{n}\right)^{T} Q_{k}\left(\check{J}_{k} \otimes I_{n}\right)<0 .
\end{aligned}
$$

For $m=2$, one obtains: $\exists P_{k} \in \mathbb{C}^{k^{2} n \times k^{2} n}, P_{k}=P_{k}^{*}>0, \exists Q_{k, 1} \in \mathbb{C}^{k^{2} n \times k^{2} n}, Q_{k, 1}=Q_{k, 1}^{*}, \exists Q_{k, 2} \in$ $\mathbb{C}^{k(k+1) n \times k(k+1) n}, Q_{k, 2}=Q_{k, 2}^{*}$,

$$
\begin{aligned}
& \left(\begin{array}{c}
\hat{J}_{k} \otimes \hat{J}_{k} \otimes I_{n} \\
\hat{J}_{k} \otimes \check{J}_{k} \otimes I_{n} \\
\check{J}_{k} \otimes \hat{J}_{k} \otimes I_{n}
\end{array}\right)^{T}\left(\begin{array}{c|c}
\left(I_{k^{2}} \otimes A_{0}\right)^{*} P_{k}+P_{k}\left(I_{k^{2}} \otimes A_{0}\right) & P_{k}\left(I_{k^{2}} \otimes A_{1}\right) P_{k}\left(I_{k^{2}} \otimes A_{2}\right) \\
\hline\left(I_{k^{2}} \otimes A_{1}\right)^{*} P_{k} & 0_{2 k^{2} n} \\
\left(I_{k^{2}} \otimes A_{2}\right)^{*} P_{k} &
\end{array}\right)\left(\begin{array}{l}
\hat{J}_{k} \otimes \hat{J}_{k} \otimes I_{n} \\
\hat{J}_{k} \otimes \check{J}_{k} \otimes I_{n} \\
\check{J}_{k} \otimes \hat{J}_{k} \otimes I_{n}
\end{array}\right) \\
& +\left(\hat{J}_{k} \otimes \hat{J}_{k} \otimes I_{n}\right)^{T} Q_{k, 1}\left(\hat{J}_{k} \otimes \hat{J}_{k} \otimes I_{n}\right)-\left(\hat{J}_{k} \otimes \check{J}_{k} \otimes I_{n}\right)^{T} Q_{k, 1}\left(\hat{J}_{k} \otimes \check{J}_{k} \otimes I_{n}\right) \\
& +\left(\hat{J}_{k} \otimes I_{k+1} \otimes I_{n}\right)^{T} Q_{k, 2}\left(\hat{J}_{k} \otimes I_{k+1} \otimes I_{n}\right)-\left(\check{J}_{k} \otimes I_{k+1} \otimes I_{n}\right)^{T} Q_{k, 2}\left(\check{J}_{k} \otimes I_{k+1} \otimes I_{n}\right)<0 .
\end{aligned}
$$

\section{Delay-independent stability of systems with noncommensurate delays and Lyapunov-Krasovskii functionals}

Consider the following delay-differential system, attached to (1)

$$
\dot{x}=A_{0} x(t)+A_{1} x\left(t-h_{1}\right)+\cdots+A_{m} x\left(t-h_{m}\right),
$$

and let $h \stackrel{\text { def }}{=} \max h_{i}$.

Definition. System (7) is said (weakly) delay-independently stable [22, 23, 24], if it is asymptotically stable for any nonnegative value of $h_{1}, \ldots, h_{m}$; that is, if

$$
\forall\left(s, h_{1}, \ldots, h_{m}\right) \in \overline{\mathbb{C}^{+}} \times[0 ;+\infty)^{m}, \operatorname{det}\left(s I_{n}-A_{0}-e^{-s h_{1}} A_{1}-\cdots-e^{-s h_{m}} A_{m}\right) \neq 0 .
$$

This property has been characterized as follows.

Theorem 2. $[\mathbf{1 9}, \mathbf{1 8}]$ System (7) is delay-independently stable, if and only if

$$
\forall\left(s, z_{1}, \ldots, z_{m}\right) \in{\overline{\mathbb{C}^{+}}} \times \overline{\mathbb{D}}^{m}, s \neq 0 \text { or } z_{1}=\cdots=z_{m}=1 \Rightarrow \operatorname{det}\left(s I_{n}-A_{0}-z_{1} A_{1}-\cdots-z_{m} A_{m}\right) \neq 0 .
$$

Extending the previous notion leads to consider

Definition. System (7) is said pointwise stable [25] or strongly delay-independently stable [30], if

$$
\forall\left(s, z_{1}, \ldots, z_{m}\right) \in{\overline{\mathbb{C}^{+}}} \overline{\mathbb{D}}^{m}, \operatorname{det}\left(s I_{n}-A_{0}-z_{1} A_{1}-\cdots-z_{m} A_{m}\right) \neq 0 .
$$


Endowing the space of the matrices $\left(A_{0}, A_{1}, \ldots, A_{m}\right)$ with the product topology induced by the maximal singular value, it is not difficult to show that the set of strongly delay-independent systems is the interior of the subspace of (weakly) delay-independent systems.

Checking delay-independent stability of system (7) is a hard task. It amounts in general to solve a NPhard problem, see $[8,37]$ and Section 6 below. Criteria based on quadratic Lyapunov-Krasovskii functionals have been obtained $[27,17,6]$. Choosing, for any $\phi \in C([-h ; 0])$ :

$$
V(\phi) \stackrel{\text { def }}{=} \phi(0)^{*} P \phi(0)+\int_{-h_{1}}^{0} \phi(\tau)^{*} Q_{1} \phi(\tau) d \tau+\cdots+\int_{-h_{m}}^{0} \phi(\tau)^{*} Q_{m} \phi(\tau) d \tau,
$$

where the matrices $P, Q_{1}, \ldots, Q_{m}$ are hermitian definite positive, yields

$$
\frac{d V\left(x_{t}\right)}{d t}=\left(\begin{array}{c}
x(t) \\
x\left(t-h_{1}\right) \\
\vdots \\
x\left(t-h_{m}\right)
\end{array}\right)^{*} R\left(\begin{array}{c}
x(t) \\
x\left(t-h_{1}\right) \\
\vdots \\
x\left(t-h_{m}\right)
\end{array}\right)
$$

with

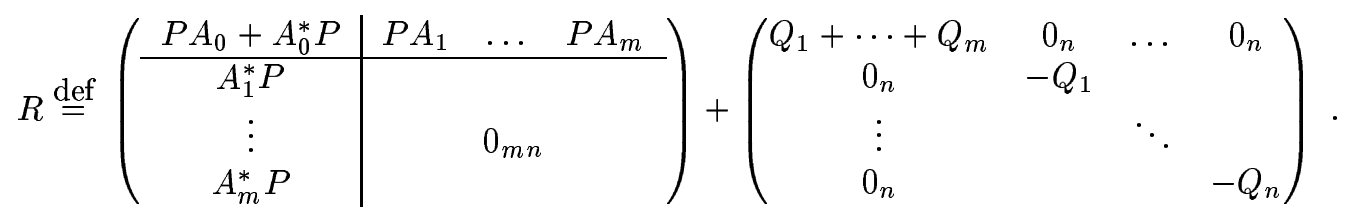

It is easily proved that solvability of the following LMI, whose unknowns are the hermitian matrices $P, Q_{1}, \ldots, Q_{m}$ :

$$
P>0, Q_{1}>0, \ldots, Q_{m}>0, R\left(P, Q_{1}, \ldots, Q_{m}\right)<0,
$$

is sufficient for delay-independent stability of (7), and even for strong delay-independent stability. However, this condition is in general not necessary [44].

We shall see now how the result in Theorem 1 is linked to the previous considerations. To each delay $h_{i}$ may be associated an operator $\nabla_{i}$, defined on the set of functions $x(t), t \in \mathbb{R}$, by: $\left(\nabla_{i} x\right)(t)=x\left(t-h_{i}\right)$. The iterates of each $\nabla_{i}$ are denoted as usual $\nabla_{i}^{k}, k \in \mathbb{N}$, in such a way that the expression $v_{k}\left(\nabla_{i}\right) x$ has a clear meaning, for $v_{k}$ defined in (4).

Now, for any $k_{1}, \ldots, k_{m} \in \mathbb{N}$, define the augmented state variable $\mathcal{X}_{k_{1}, \ldots, k_{m}}(t) \in \mathbb{C}^{k_{1} \ldots k_{m} n}$ by:

$$
\mathcal{X}_{k_{1}, \ldots, k_{m}} \stackrel{\text { def }}{=} v_{k_{m}}\left(\nabla_{m}\right) \otimes \cdots \otimes v_{k_{1}}\left(\nabla_{1}\right) x .
$$

As an example, for two delays, vector $\mathcal{X}_{k_{1}, k_{2}}(t)$ is constituted by the concatenation of the $k_{2}$ vectors of $\mathbb{C}^{k_{1} n}$

$$
\left(\begin{array}{c}
x(t) \\
x\left(t-h_{1}\right) \\
\vdots \\
x\left(t-\left(k_{1}-1\right) h_{1}\right)
\end{array}\right),\left(\begin{array}{c}
x\left(t-h_{2}\right) \\
x\left(t-h_{2}-h_{1}\right) \\
\vdots \\
x\left(t-h_{2}-\left(k_{1}-1\right) h_{1}\right)
\end{array}\right), \ldots \text { until }\left(\begin{array}{c}
x\left(t-\left(k_{2}-1\right) h_{2}\right) \\
x\left(t-\left(k_{2}-1\right) h_{2}-h_{1}\right) \\
\vdots \\
x\left(t-\left(k_{2}-1\right) h_{2}-\left(k_{1}-1\right) h_{1}\right)
\end{array}\right) .
$$

With this notation, the functional (8) may be written

$$
\mathcal{X}_{1, \ldots, 1}(t)^{*} P \mathcal{X}_{1, \ldots, 1}(t)+\sum_{i=1}^{m} \int_{t-h_{i}}^{t} \mathcal{X}_{1, \ldots, 1}(\tau)^{*} Q_{i} \mathcal{X}_{1, \ldots, 1}(\tau) d \tau
$$

Consider instead, along the trajectories of (7), the following functional:

$$
\begin{aligned}
& V_{k}\left(\left(\mathcal{X}_{k+1, \ldots, k+1, k}\right)_{t}\right) \stackrel{\text { def }}{=} \mathcal{X}_{k, \ldots, k}(t)^{*} P_{k} \mathcal{X}_{k, \ldots, k}(t)+\int_{t-h_{1}}^{t} \mathcal{X}_{k, \ldots, k}(\tau)^{*} Q_{k, 1} \mathcal{X}_{k, \ldots, k}(\tau) d \tau \\
& \quad+\int_{t-h_{2}}^{t} \mathcal{X}_{k+1, k, \ldots, k}(\tau)^{*} Q_{k, 2} \mathcal{X}_{k+1, k, \ldots, k}(\tau) d \tau+\cdots+\int_{t-h_{m}}^{t} \mathcal{X}_{k+1, \ldots, k+1, k}(\tau)^{*} Q_{k, m} \mathcal{X}_{k+1, \ldots, k+1, k}(\tau) d \tau .
\end{aligned}
$$

RR $n^{\circ} 4316$ 
The previous functional is parametrized by the $(m+1)$ hermitian matrices $P_{k} \in \mathbb{C}^{k^{m} n \times k^{m} n}, Q_{k, i} \in$ $\mathbb{C}^{k^{m-i+1}(k+1)^{i-1} n \times k^{m-i+1}(k+1)^{i-1} n}, i=1 \ldots m$. The value of $V_{k}$ at time $t$ depends only upon the values of $x$ on the interval $[t-k m h ; t]$.

It turns out that

$$
\frac{d\left[V_{k}\left(\left(\mathcal{X}_{k+1, \ldots, k+1, k}\right)_{t}\right)\right]}{d t}=\mathcal{X}_{k+1, \ldots, k+1}(t)^{*} R_{k}^{\mathbb{C}} \mathcal{X}_{k+1, \ldots, k+1}(t)
$$

where $R_{k}^{\mathbb{C}}$ is defined by (5). The proof of this is not difficult, and is left to the reader. It relates essentially to the fact that

$$
\hat{J}_{k} v_{k+1}\left(\nabla_{i}\right) x=v_{k}\left(\nabla_{i}\right) x, \check{J}_{k} v_{k+1}\left(\nabla_{i}\right) x=\nabla_{i}\left(v_{k}\left(\nabla_{i}\right) x\right) .
$$

These identities will be used latter under a different, frequency domain, form, see formula (13) below.

In particular, if $\left(P_{k}, Q_{k, 1}, \ldots, Q_{k, m}\right)$ is a solution of $\mathrm{LMI}_{k}^{\mathbb{C}}$ such that the matrices $Q_{k, 1}, \ldots, Q_{k, m}$ are definite positive, then $V_{k}$ is a Lyapunov-Krasovskii functional for system (7), for any nonnegative values of the delays $h_{1}, \ldots, h_{m}$.

For the case of a unique delay $(m=1)$, solvability of $\mathrm{LMI}_{k}^{\mathbb{C}}$ is equivalent to solvability by a solution $\left(P_{k}, Q_{k}\right)$ with $Q_{k}>0$, see [2]. In other words, all the systems in a sufficiently small neighborhood of system

$$
\dot{x}=A_{0} x(t)+A_{1} x(t-h)
$$

are delay-independently stable, if and only if there exist a positive integer $k$ and two hermitian matrices $P_{k}, Q_{k}$ in $\mathbb{C}^{k n \times k n}$, such that

$$
P_{k}>0, Q_{k}>0, R_{k}^{\mathbb{C}}=\left(\begin{array}{cc}
I_{k n} & 0_{k n \times n} \\
0_{k n \times n} & I_{k n}
\end{array}\right)^{T}\left(\begin{array}{cc}
P_{k}\left(I_{k} \otimes A_{0}\right)+\left(I_{k} \otimes A_{0}\right)^{*} P_{k} & P_{k}\left(I_{k} \otimes A_{1}\right) \\
\left(I_{k} \otimes A_{1}\right)^{*} P_{k} & 0_{k n}
\end{array}\right)\left(\begin{array}{cc}
I_{k n} & 0_{k n \times n} \\
0_{k n \times n} & I_{k n}
\end{array}\right)<0,
$$

that is, if and only if these systems admit a definite positive quadratic Lyapunov-Krasovskii functional of the type

$$
\left(\begin{array}{c}
x(t) \\
x(t-h) \\
\vdots \\
x(t-(k-1) h)
\end{array}\right)^{*} P_{k}\left(\begin{array}{c}
x(t) \\
x(t-h) \\
\vdots \\
x(t-(k-1) h)
\end{array}\right)+\int_{t-h}^{t}\left(\begin{array}{c}
x(\tau) \\
x(\tau-h) \\
\vdots \\
x(\tau-(k-1) h)
\end{array}\right)^{*} Q_{k}\left(\begin{array}{c}
x(\tau) \\
x(\tau-h) \\
\vdots \\
x(\tau-(k-1) h)
\end{array}\right) d \tau
$$

independent upon the (nonnegative) values of $h$, and decreasing along the trajectories of (10). We conjecture that the same remains true for $m>1$. From the numerical point of view, however, no time gain may be expected when adding supplementary constraint in the LMIs.

\section{$5 \quad$ Robust stability of systems with real parameters}

Theorem 3. The matrix $A_{0}+r_{1} A_{1}+\cdots+r_{m} A_{m}$ is Hurwitz for any $r_{1}, \ldots, r_{m} \in[-1 ;+1]$, if and only if there exist a positive integer $k$ and $(m+1)$ hermitian matrices

$$
P_{k} \in \mathbb{C}^{k^{m} n \times k^{m} n} \text { and } Q_{k, i} \in \mathbb{C}^{k^{m-i+1}(k+1)^{i-1} n \times k^{m-i+1}(k+1)^{i-1} n}, i=1 \ldots m,
$$

such that the following LMI is solvable:

$$
P_{k}>0, R_{k}^{\mathbb{R}}\left(P_{k}, Q_{k, 1}, \ldots, Q_{k, m}\right)<0,
$$


where $R_{k}^{\mathbb{R}} \in \mathbb{C}^{(k+1)^{m} n \times(k+1)^{m} n}$ is defined as

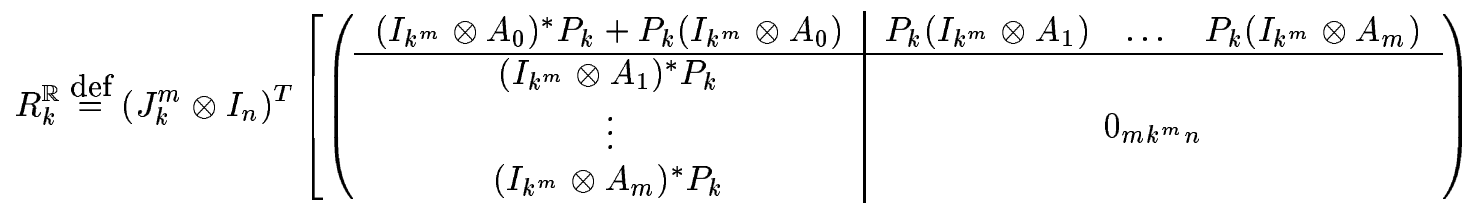

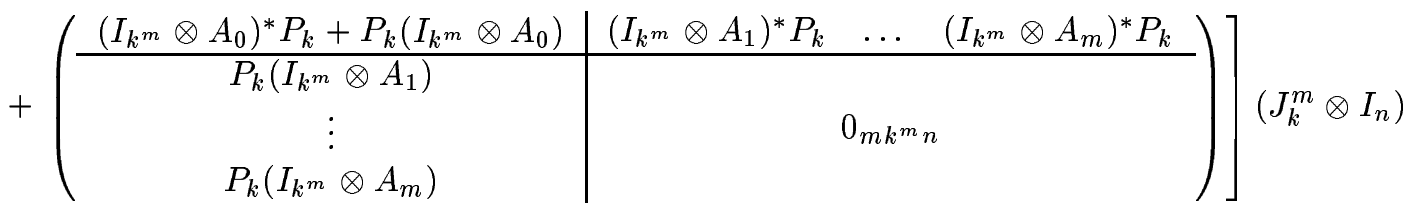

$$
\begin{aligned}
& +2 \sum_{i=1}^{m}\left(\left(\hat{J}_{k, i}^{m} \otimes I_{n}\right)^{T} Q_{k, i}\left(\hat{J}_{k, i}^{m} \otimes I_{n}\right)-\left(\check{J}_{k, i}^{m} \otimes I_{n}\right)^{T} Q_{k, i}\left(\check{J}_{k, i}^{m} \otimes I_{n}\right)\right) .
\end{aligned}
$$

Moreover,

- if $\mathrm{LMI}_{k}^{\mathbb{R}}$ is solvable, then it is also solvable for indexes $k^{\prime} \geq k$;

- if the matrices $A_{i}, 0 \leq i \leq m$, are real, then the statement holds with real, symmetric, matrices $P_{k}, Q_{k, i}$, $1 \leq i \leq m$.

Proof of Theorem 3 is given in Section 8.3.

For $m=1$ and $m=2$ respectively, the two following families of LMIs are obtained: $\exists P_{k} \in \mathbb{C}^{k n \times k n}, P_{k}=$ $P_{k}^{*}>0, \exists Q_{k} \in \mathbb{C}^{k n \times k n}, Q_{k}=Q_{k}^{*}$,

$$
\begin{array}{r}
\left(\begin{array}{c}
\hat{J}_{k} \otimes I_{n} \\
\tilde{J}_{k} \otimes I_{n}
\end{array}\right)^{T}\left(\begin{array}{cc}
\left(I_{k} \otimes A_{0}\right)^{*} P_{k}+P_{k}\left(I_{k} \otimes A_{0}\right) & \frac{1}{2}\left(P_{k}\left(I_{k} \otimes A_{1}\right)+\left(I_{k} \otimes A_{1}\right)^{*} P_{k}\right) \\
\frac{1}{2}\left(P_{k}\left(I_{k} \otimes A_{1}\right)+\left(I_{k} \otimes A_{1}\right)^{*} P_{k}\right) & 0_{k n}
\end{array}\right)\left(\begin{array}{l}
\hat{J}_{k} \otimes I_{n} \\
\check{J}_{k} \otimes I_{n}
\end{array}\right) \\
+\left(\hat{J}_{k} \otimes I_{n}\right)^{T} Q_{k}\left(\hat{J}_{k} \otimes I_{n}\right)-\left(\check{J}_{k} \otimes I_{n}\right)^{T} Q_{k}\left(\check{J}_{k} \otimes I_{n}\right)<0,
\end{array}
$$

and: $\exists P_{k} \in \mathbb{C}^{k^{2} n \times k^{2} n}, P_{k}=P_{k}^{*}>0, \exists Q_{k, 1} \in \mathbb{C}^{k^{2} n \times k^{2} n}, Q_{k, 1}=Q_{k, 1}^{*}, \exists Q_{k, 2} \in \mathbb{C}^{k(k+1) n \times k(k+1) n}, Q_{k, 2}=Q_{k, 2}^{*}$,

$$
\begin{aligned}
& \left(\begin{array}{c|c}
\hat{J}_{k} \otimes \hat{J}_{k} \otimes I_{n} \\
\hat{J}_{k} \otimes \breve{J}_{k} \otimes I_{n} \\
\tilde{J}_{k} \otimes \hat{J}_{k} \otimes I_{n}
\end{array}\right)^{T}\left[\left(\begin{array}{c|c}
\left(I_{k^{2}} \otimes A_{0}\right)^{*} P_{k}+P_{k}\left(I_{k^{2}} \otimes A_{0}\right) & P_{k}\left(I_{k^{2}} \otimes A_{1}\right) P_{k}\left(I_{k^{2}} \otimes A_{2}\right) \\
\hline\left(I_{k^{2}} \otimes A_{1}\right)^{*} P_{k} & 0_{2 k^{2} n} \\
\left(I_{k^{2}} \otimes A_{2}\right)^{*} P_{k} &
\end{array}\right)\right. \\
& \left.\left(\begin{array}{c|cc}
\left(I_{k^{2}} \otimes A_{0}\right)^{*} P_{k}+P_{k}\left(I_{k^{2}} \otimes A_{0}\right) & \left(I_{k^{2}} \otimes A_{1}\right)^{*} P_{k} & \left(I_{k^{2}} \otimes A_{2}\right)^{*} P_{k} \\
\hline P_{k}\left(I_{k^{2}} \otimes A_{1}\right) & 0_{2 k^{2} n}
\end{array}\right)\right]\left(\begin{array}{c}
\hat{J}_{k} \otimes \hat{J}_{k} \otimes I_{n} \\
P_{k}\left(I_{k^{2}} \otimes A_{2}\right)
\end{array}\right. \\
& +2\left(\left(\hat{J}_{k} \otimes \hat{J}_{k} \otimes I_{n}\right)^{T} Q_{k, 1}\left(\hat{J}_{k} \otimes \hat{J}_{k} \otimes I_{n}\right)-\left(\hat{J}_{k} \otimes \check{J}_{k} \otimes I_{n}\right)^{T} Q_{k, 1}\left(\hat{J}_{k} \otimes \check{J}_{k} \otimes I_{n}\right)\right) \\
& +2\left(\left(\hat{J}_{k} \otimes I_{k+1} \otimes I_{n}\right)^{T} Q_{k, 2}\left(\hat{J}_{k} \otimes I_{k+1} \otimes I_{n}\right)-\left(\check{J}_{k} \otimes I_{k+1} \otimes I_{n}\right)^{T} Q_{k, 2}\left(\check{J}_{k} \otimes I_{k+1} \otimes I_{n}\right)\right)<0 .
\end{aligned}
$$

Theorems 1 and 3 are easily adapted to treat the mixed complex/real case. The result is not stated completely here, for sake of space. As an example, for stability analysis of $A_{0}+z A_{1}+r A_{2}$, for a complex parameter $z$ and a real parameter $r$, the criterion is based on the families of LMIs: $\exists P_{k} \in \mathbb{C}^{k^{2} n \times k^{2} n}, P_{k}=$ $P_{k}^{*}>0, \exists Q_{k, 1} \in \mathbb{C}^{k^{2} n \times k^{2} n}, Q_{k, 1}=Q_{k, 1}^{*}, \exists Q_{k, 2} \in \mathbb{C}^{k(k+1) n \times k(k+1) n}, Q_{k, 2}=Q_{k, 2}^{*}$,

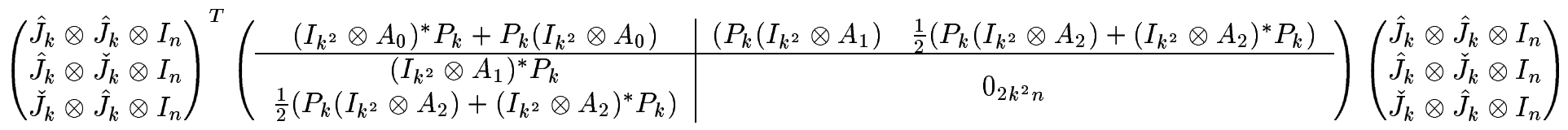

$$
\begin{aligned}
& +\left(\hat{J}_{k} \otimes \hat{J}_{k} \otimes I_{n}\right)^{T} Q_{k, 1}\left(\hat{J}_{k} \otimes \hat{J}_{k} \otimes I_{n}\right)-\left(\hat{J}_{k} \otimes \check{J}_{k} \otimes I_{n}\right)^{T} Q_{k, 1}\left(\hat{J}_{k} \otimes \check{J}_{k} \otimes I_{n}\right) \\
& +\left(\hat{J}_{k} \otimes I_{k+1} \otimes I_{n}\right)^{T} Q_{k, 2}\left(\hat{J}_{k} \otimes I_{k+1} \otimes I_{n}\right)-\left(\check{J}_{k} \otimes I_{k+1} \otimes I_{n}\right)^{T} Q_{k, 2}\left(\check{J}_{k} \otimes I_{k+1} \otimes I_{n}\right)<0 .
\end{aligned}
$$

$\mathrm{RR} \mathrm{n}^{\circ} 4316$ 


\section{Complexity issues}

Robust stability may be expressed in terms of structured singular values [12, 45]. For this, define the square matrix $M(s) \in \mathbb{C}^{m n \times m n}$ by

$$
M(s) \stackrel{\text { def }}{=}\left(\begin{array}{c}
I_{n} \\
\vdots \\
I_{n}
\end{array}\right)\left(s I-A_{0}\right)^{-1}\left(\begin{array}{lll}
A_{1} & \ldots & A_{m}
\end{array}\right)
$$

and the uncertainty set $\Delta$ as

$$
\Delta=\left\{\operatorname{diag}\left\{z_{1} I_{n} ; \ldots ; z_{m} I_{n}\right\}: z_{i} \in \mathbb{C}\right\} .
$$

The key result is borrowed to Chen et al.

Theorem 4. [8] System (7) is delay-independently stable, if and only if the three following conditions are fulfilled:

- $A_{0}$ is asymptotically stable,

- $\forall s \in j \mathbb{R} \backslash\{0\}, \mu_{\Delta}(M(s))<1$,

- $\operatorname{det}\left(I_{m n}-M(0)\right) \neq 0$.

System (7) is strongly delay-independently stable, or equivalently system (1) is asymptotically stable for any $z_{1}, \ldots, z_{m}$ in $\overline{\mathbb{D}}$, if and only if the two following conditions are fulfilled:

- $A_{0}$ is asymptotically stable,

- $\forall s \in j \mathbb{R}, \mu_{\Delta}(M(s))<1$.

Similarly, stability of $(1)$ for any real value of $z_{1}, \ldots, z_{m}$ in $[-1 ;+1]$, may be characterized with the corresponding set $\Delta$ of real uncertainties.

Using various $\mu$ approximations, the interpretation furnished by Theorem 4 permits to obtain robust stability criteria. As an example, the method described in Section 4 and leading to LMI (9), results in limitation to constant scalings when evaluating $\mu$, see [44].

Complexity issues associated to evaluation of the structured singular values have been studied. It has been proved by Braatz et al. [7] that this problem is NP-hard, and by Toker et al. [38] that the same holds for the purely complex $\mu$. The simpler problem of determining whether a delay system is delay-independently stable is NP-hard too, see [37]. Moreover, the real $\mu$ has been proved hardly approximable by Fu in [14], and Treil has announced and proved that "the gap between complex structured singular value $\mu$ and its upper bound is infinite", see $[39,36]$.

The results of Theorems 1 and 3 offer attractive numerical alternative for the evaluation of certain structured singular values, as the resolution of LMIs is a convex problem, solvable in polynomial time. They furnishe approximations by families of $\mathrm{P}$ problems, of arbitrary precision, for a class of NP problems. Up to our knowledge, this point is original. Moreover, the LMIs involved are determined simply, by elementary algebraic operations.

In their present state, the main weakness of the results is non-decidibility: no information is known on the size of the least $k$, if any, for which the LMIs are solvable (in other words, of the largest $k$ which is necessary to test numerically to decide whether the system is robustly stable or not). This is an important question, both from theoretical and practical point of view. Numerical experiments indicate that small values of $k$ often yield correct answers. On the other hand, suppose there exists a bound $k(n, m)$, such that, for any system of size $n$ comprising $m$ parameters, robust stability is equivalent to solvability of the associated LMI for $k=k(n, m)$. Then the growth at infinity of $k(n, m)$ is larger than any power of $n$, otherwise $\mathrm{NP}=\mathrm{P}$. Effective use of large values of $k$ hinges upon the possibility of intensive computation. 


\section{Numerical example}

Let $n=3$,

$$
A_{0}=\left(\begin{array}{ccc}
-12 & -7 & 7 \\
-11 & -13 & -5 \\
-2 & 9 & -8
\end{array}\right), A_{1}=\left(\begin{array}{lll}
0 & 1 & 0 \\
1 & 0 & 2 \\
0 & 3 & 0
\end{array}\right), A_{2}=\left(\begin{array}{ccc}
1 & 2 & 0 \\
-3 & -1 & 0 \\
-1 & 0 & 0
\end{array}\right)
$$

We shall evaluate the following robustness margins:

$$
\begin{aligned}
& \alpha_{z z} \stackrel{\text { def }}{=} \sup _{\left(z_{1}, z_{2}\right) \in \overline{\mathbb{D}}^{2}} \operatorname{Re} \sigma\left(A_{0}+z_{1} A_{1}+z_{2} A_{2}\right), \\
& \alpha_{z r} \stackrel{\text { def }}{=} \sup _{\left(z_{1}, r_{2}\right) \in \overline{\mathbb{D}} \times[-1 ;+1]} \operatorname{Re} \sigma\left(A_{0}+z_{1} A_{1}+r_{2} A_{2}\right), \\
& \alpha_{r z} \stackrel{\text { def }}{=} \sup _{\left(r_{1}, z_{2}\right) \in[-1 ;+1] \times \overline{\mathbb{D}}} \operatorname{Re} \sigma\left(A_{0}+r_{1} A_{1}+z_{2} A_{2}\right), \\
& \alpha_{z} \stackrel{\text { def }}{=} \sup _{z \in \overline{\mathbb{D}}} \operatorname{Re} \sigma\left(A_{0}+z\left(A_{1}+A_{2}\right)\right), \\
& \alpha_{r r} \stackrel{\text { def }}{=} \sup _{\left(r_{1}, r_{2}\right) \in[-1 ;+1]^{2}} \operatorname{Re} \sigma\left(A_{0}+r_{1} A_{1}+r_{2} A_{2}\right), \\
& \alpha_{r} \stackrel{\text { deff }}{=} \sup _{r \in[-1 ;+1]} \operatorname{Re} \sigma\left(A_{0}+r\left(A_{1}+A_{2}\right)\right) .
\end{aligned}
$$

Clearly, the latter quantities are linked by the inequalities:

$$
\alpha_{z z} \geq \alpha_{z r}, \alpha_{r z} \geq \alpha_{r r} \geq \alpha_{r}, \text { and } \alpha_{z z} \geq \alpha_{z} \geq \alpha_{r} .
$$

We use the LMIs previously presented, to find, for each uncertainty structure, the smallest real number $\alpha$ such that $A_{0}-\alpha I_{n}+z_{1} A_{1}+z_{2} A_{2}$ is robustly stable. For each integer $k$ and each value of $\alpha$, a convex problem is solved, but the problem is not jointly convex in the four unknowns $P_{k}, Q_{k, 1}, Q_{k, 2}$ and $\alpha$, so a dichotomy process is achieved.

The computations presented here have been achieved using the package lmitool of the free software ScILAB. The successive (upper) estimates of the robustness margins, according to the value of $k$, are given in Table 1. Between parentheses is given the CPU time necessary for resolution of the LMIs (for the corresponding values of $\alpha$ and $k$ ), measured on a computer equipped with a Pentium III $800 \mathrm{MHz}$.

\begin{tabular}{c|c|c|c|c|c|c|} 
& $\alpha_{z z}$ & $\alpha_{z r}$ & $\alpha_{r z}$ & $\alpha_{z}$ & $\alpha_{r r}$ & $\alpha_{r}$ \\
\hline \hline$k=1$ & $-2.42(0.2 \mathrm{~s})$ & $-3.17(0.2 \mathrm{~s})$ & $-2.42(0.19 \mathrm{~s})$ & $-3.24(0.06 \mathrm{~s})$ & $-3.17(0.2 \mathrm{~s})$ & $-3.24(0.07 \mathrm{~s})$ \\
$k=2$ & $-3.87(7.63 \mathrm{~s})$ & $-4.57(9.73 \mathrm{~s})$ & $-4.46(7.84 \mathrm{~s})$ & $-4.14(0.23 \mathrm{~s})$ & $-5.24(10.1 \mathrm{~s})$ & $-5.39(0.26 \mathrm{~s})$ \\
$k=3$ & $-3.87(5 \min 03 \mathrm{~s})$ & $-4.57(6 \min 19 \mathrm{~s})$ & $-4.46(5 \min 10 \mathrm{~s})$ & $-4.14(0.63 \mathrm{~s})$ & $-5.24(6 \min 29 \mathrm{~s})$ & $-5.41(0.65 \mathrm{~s})$
\end{tabular}

Table 1: Succesive estimates of the margins and corresponding CPU times.

One verifies that, for each margin, the successive estimates are nonincreasing functions of $k$, and that the inequalities corresponding to (12) are fulfilled for any value of $k$. The true values of the margins (up to the precision considered) have been computed by gridding. They are attained for values of the parameters indicated in Table 2. In the present case, the tests achieved for $k=2$ provide these true values up to three digits, except for $\alpha_{r}(k=3)$. 


\begin{tabular}{|c|c|c|c|c|c|}
$\alpha_{z z}$ & $\alpha_{z r}$ & $\alpha_{r z}$ & $\alpha_{z}$ & $\alpha_{r r}$ & $\alpha_{r}$ \\
\hline$z_{1}=e^{0.72 i \pi}$ & $z_{1}=e^{0.7 i \pi}$ & $r_{1}=-1$ & $z=e^{0.57 i \pi}$ & $r_{1}=-1$ & $r=1$ \\
$z_{2}=e^{0.43 i \pi}$ & $r_{2}=0.9$ & $z_{2}=e^{0.45 i \pi}$ & & $r_{2}=0.5$ &
\end{tabular}

Table 2: Value of $\left(z_{1}, z_{2}\right)$ realizing the maximum of the largest real part in the spectrum of $\left(A_{0}+z_{1} A_{1}+z_{2} A_{2}\right)$, for each margin.

\section{Proofs}

\subsection{Additional notations and preparation}

With $v_{k}$ as in (4), one shows easily that

$$
\hat{J}_{k} v_{k+1}(z)=v_{k}(z), \check{J}_{k} v_{k+1}(z)=z v_{k}(z) .
$$

Other elementary properties are the following: $\forall p, q \in \mathbb{N}, \forall M \in \mathbb{C}^{p \times q}$,

$$
\begin{gathered}
\left(v_{k}(z) \otimes I_{p}\right) M=\left(I_{k} \otimes M\right)\left(v_{k}(z) \otimes I_{q}\right), \\
\left(v_{k}\left(z_{m}\right) \otimes \cdots \otimes v_{k}\left(z_{1}\right) \otimes I_{p}\right)=\left(v_{k}\left(z_{m}\right) \otimes \cdots \otimes v_{k}\left(z_{2}\right) \otimes I_{k p}\right)\left(v_{k}\left(z_{1}\right) \otimes I_{p}\right), \\
\left\|v_{k}\left(z_{1}\right)\right\|^{2} I_{p}=\left(v_{k}\left(z_{1}\right) \otimes I_{p}\right)^{*}\left(v_{k}\left(z_{1}\right) \otimes I_{p}\right) .
\end{gathered}
$$

From (13), (14), the following result is deduced.

Lemma 5. For any $k, i, m, n \in \mathbb{N}, 1 \leq i \leq m$, the following identities hold:

$$
\begin{aligned}
& \left(\begin{array}{cccc}
\left(v_{k}(z) \otimes I_{k^{m} n}\right) & 0 & \cdots & 0 \\
0 & \left(v_{k}(z) \otimes I_{k^{m} n}\right) & \cdots & 0 \\
\vdots & \vdots & \ddots & \vdots \\
0 & 0 & \ldots & \left(v_{k}(z) \otimes I_{k^{m} n}\right)
\end{array}\right)\left(J_{k}^{m} \otimes I_{n}\right)=\left(J_{k}^{m+1} \otimes I_{n}\right)\left(v_{k+1}(z) \otimes I_{(k+1)^{m} n}\right), \\
& \left(v_{k}(z) \otimes I_{k^{m-i+1}(k+1)^{i-1} n}\right)\left(\hat{J}_{k, i}^{m} \otimes I_{n}\right)=\left(\hat{J}_{k, i}^{m+1} \otimes I_{n}\right)\left(v_{k+1}(z) \otimes I_{(k+1)^{m} n}\right), \\
& \left(v_{k}(z) \otimes I_{k^{m-i+1}(k+1)^{i-1} n}\right)\left(\check{J}_{k, i}^{m} \otimes I_{n}\right)=\left(\check{J}_{k, i}^{m+1} \otimes I_{n}\right)\left(v_{k+1}(z) \otimes I_{(k+1)^{m} n}\right) .
\end{aligned}
$$

Proof. Identity (17b) is obtained by the following sequence of calculus.

$$
\begin{aligned}
\left(v_{k}(z) \otimes I_{k^{m-i+1}(k+1)^{i-1} n}\right)\left(\hat{J}_{k, i}^{m} \otimes I_{n}\right) & \left.=\left(\hat{J}_{k} v_{k+1}(z) \otimes I_{k^{m-i+1}(k+1)^{i-1} n}\right)\left(\hat{J}_{k, i}^{m} \otimes I_{n}\right) \quad \text { (by }(13)\right) \\
& =\left(\hat{J}_{k} \otimes I_{k^{m-i+1}(k+1)^{i-1} n}\right)\left(v_{k+1}(z) \otimes I_{k^{m-i+1}(k+1)^{i-1} n}\right)\left(\hat{J}_{k, i}^{m} \otimes I_{n}\right) \\
& =\left(\hat{J}_{k} \otimes I_{k^{m-i+1}(k+1)^{i-1} n}\right)\left(I_{k} \otimes \hat{J}_{k, i}^{m} \otimes I_{n}\right)\left(v_{k+1}(z) \otimes I_{(k+1)^{m} n}\right) \\
& =\left(\hat{J}_{k} \otimes \hat{J}_{k, i}^{m} \otimes I_{n}\right)\left(v_{k+1}(z) \otimes I_{(k+1)^{m} n}\right) \\
& =\left(\hat{J}_{k, i}^{m+1} \otimes I_{n}\right)\left(v_{k+1}(z) \otimes I_{(k+1)^{m} n}\right) \quad(\text { by }(3 \mathrm{~b})) .
\end{aligned}
$$

Identities (17a) and (17c) are proved by similar arguments, based on (3a), (3c).

Last, we shall use for the matrix $\hat{J}_{k}$ defined in Section 2 , the following block decomposition:

$$
\hat{J}_{k}=\left(\begin{array}{ll}
I_{k} & 0_{k \times 1}
\end{array}\right)=\left(\begin{array}{ll}
f_{k} & F_{k}
\end{array}\right),
$$

and the spectrum of $F_{k}$ is $\{0\}$. Simple computation shows that $f_{k}=\left(I_{k}-z F_{k}\right) v_{k}(z)$, that is

$$
\left(I_{k}-z F_{k}\right)^{-1} f_{k}=v_{k}(z) \text {. }
$$

Another useful property is the fact that, for any $i \in \mathbb{N}, 0 \leq i \leq k-1$,

$$
F_{k}^{i T} v_{k}(z)=z^{i}\left(\begin{array}{c}
v_{k-i}(z) \\
0_{i \times 1}
\end{array}\right)
$$




\subsection{Proof of Theorem 1}

Consider the parameter-dependent Lyapunov inequality

$$
P\left(z_{1}, \ldots, z_{m}\right)\left(A_{0}+z_{1} A_{1}+\cdots+z_{m} A_{m}\right)+\left(A_{0}+z_{1} A_{1}+\cdots+z_{m} A_{m}\right)^{*} P\left(z_{1}, \ldots, z_{m}\right)<0 .
$$

The principle of the proof of Theorem 1 lies in the following steps.

$$
\begin{aligned}
& \forall\left(z_{1}, \ldots, z_{m}\right) \in \overline{\mathbb{D}}^{m}, A_{0}+z_{1} A_{1}+\cdots+z_{m} A_{m} \text { is Hurwitz, } \\
& \Rightarrow \forall\left(z_{1}, \ldots, z_{m}\right) \in \overline{\mathbb{D}}^{m}, \exists P\left(z_{1}, \ldots, z_{m}\right)=P\left(z_{1}, \ldots, z_{m}\right)^{*}>0 \text { fulfilling (21), } \\
& \Rightarrow \quad \exists P\left(z_{1}, \ldots, z_{m}\right)=P\left(z_{1}, \ldots, z_{m}\right)^{*}>0 \text { analytic wrt } z_{1}, \ldots, z_{m}, z_{1}^{*}, \ldots, z_{m}^{*} \text { in } \overline{\mathbb{D}}^{m} \text {, fulfilling (21), } \\
& \Rightarrow \exists \bar{k} \in \mathbb{N}, \forall k \geq \bar{k}, \exists P\left(z_{1}, \ldots, z_{m}\right)=P\left(z_{1}, \ldots, z_{m}\right)^{*}>0 \text { polynomial of degree } k-1 \text { wrt } z_{1}, \ldots, z_{m} \text {, } \\
& z_{1}^{*}, \ldots, z_{m}^{*} \text { in } \overline{\mathbb{D}}^{m} \text {, fulfilling (21), } \\
& \Rightarrow \quad \exists \bar{k} \in \mathbb{N}, \forall k \geq \bar{k}, \exists P_{k} \in \mathbb{C}^{k^{m} n \times k^{m} n}, P_{k}=P_{k}^{*}>0, \forall\left(z_{1}, \ldots, z_{m}\right) \in \overline{\mathbb{D}}^{m}, P\left(z_{1}, \ldots, z_{m}\right) \text { fulfills (21), with } \\
& P\left(z_{1}, \ldots, z_{m}\right) \stackrel{\text { def }}{=} \frac{1}{\left\|v_{k}\left(z_{1}\right)\right\|^{2} \ldots\left\|v_{k}\left(z_{m}\right)\right\|^{2}}\left(v_{k}\left(z_{1}\right) \otimes \cdots \otimes v_{k}\left(z_{m}\right) \otimes I_{n}\right)^{*} P_{k}\left(v_{k}\left(z_{1}\right) \otimes \cdots \otimes v_{k}\left(z_{m}\right) \otimes I_{n}\right), \\
& \Rightarrow \quad \exists \bar{k} \in \mathbb{N}, \forall k \geq \bar{k}, \operatorname{LMI}_{k}^{\mathbb{C}} \text { is solvable (same } \bar{k} \text { and } P_{k} \text { than in (22e)) } \\
& \Rightarrow \quad \exists \bar{k} \in \mathbb{N}, \forall k \geq \bar{k}, \exists P_{k} \in \mathbb{C}^{k^{m} n \times k^{m} n}, P_{k}=P_{k}^{*}>0 \text { (same } \bar{k} \text { and } P_{k} \text { than in (22e)), such that, } \\
& \forall\left(z_{1}, \ldots, z_{m}\right) \in(\partial \mathbb{D})^{m}, P(z) \text { as in (22e) fulfills (21), } \\
& \Rightarrow \quad \forall\left(z_{1}, \ldots, z_{m}\right) \in(\partial \mathbb{D})^{m}, \exists P\left(z_{1}, \ldots, z_{m}\right)=P\left(z_{1}, \ldots, z_{m}\right)^{*}>0 \text { fulfilling (21), } \\
& \Rightarrow \quad(22 \mathrm{a}) \text {. }
\end{aligned}
$$

From the previous set of implications, one obtains the equivalence (22a) $\Leftrightarrow(22 \mathrm{f})$, which is the first result in Theorem 1. The fact that solvability of the $\mathrm{LMI}_{k}^{\mathbb{C}}$ implies the same property for largest ranks is also deduced. Last, when the coefficients are real, then the analytic and polynomial solutions exhibited above are easily proved to be real too.

It now remains to prove the above relations. The implications $(22 \mathrm{a}) \Rightarrow(22 \mathrm{~b})$ and $(22 \mathrm{~g}) \Rightarrow(22 \mathrm{~h})$ are straightforward. Let us establish the other ones.

\subsubsection{Proof of $(22 b) \Rightarrow(22 c)$.}

This implication may be proved using the results by Delchamps [9] on regularity of the solutions of Riccati equation wrt coefficients, results generalized by Kamen in [26, Chapter 4, Lemma p. 134] to complex analyticity. They ensure that $P\left(z_{1}, \ldots z_{m}\right)$, the solution of the parameter-dependent Lyapunov equation

$$
P\left(z_{1}, \ldots, z_{m}\right)\left(A_{0}+z_{1} A_{1}+\cdots+z_{m} A_{m}\right)+\left(A_{0}+z_{1} A_{1}+\cdots+z_{m} A_{m}\right)^{*} P\left(z_{1}, \ldots, z_{m}\right)=-I_{n},
$$

is analytic in $z_{i}$ and $z_{i}^{* 1}$. This may also be seen from the explicit form of the solution for the previous identity, namely:

$$
P\left(z_{1}, \ldots, z_{m}\right)=\int_{0}^{+\infty} e^{\left(A_{0}+z_{1} A_{1}+\cdots+z_{m} A_{m}\right)^{*} t} e^{\left(A_{0}+z_{1} A_{1}+\cdots+z_{m} A_{m}\right) t} d t
$$

on expanding the integrand in powers of $z_{i}, z_{i}^{*}, 1 \leq i \leq m$, and using the uniformity of the convergence in $t=+\infty$ wrt the $z_{i}$ in the product of closed unit balls. In other words, there exists a family of hermitian

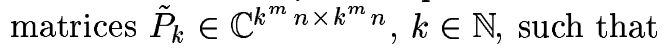

$$
P\left(z_{1}, \ldots, z_{m}\right)=\lim _{k \rightarrow+\infty}\left(v_{k}\left(z_{m}\right) \otimes \cdots \otimes v_{k}\left(z_{1}\right) \otimes I_{n}\right)^{*} \tilde{P}_{k}\left(v_{k}\left(z_{m}\right) \otimes \cdots \otimes v_{k}\left(z_{1}\right) \otimes I_{n}\right),
$$

in the sense of uniform convergence in $\overline{\mathbb{D}}^{m}$. In the previous expression, the matrix $\tilde{P}_{k}$ defines the value of those terms of the expansion whose partial degree in $z_{i}$ and $z_{i}^{*}$ is less than or equal to $k-1$ for any $1 \leq i \leq m$.

\footnotetext{
${ }^{1}$ More precisely, there exists a function $f$ analytic in $\overline{\mathbb{D}}^{2 m}$ such that $P\left(z_{1}, \ldots, z_{m}\right)=f\left(z_{1}, \ldots, z_{m}, z_{1}^{*}, \ldots, z_{m}^{*}\right)$. This precision is omitted in the sequel.

$\mathrm{RR} \mathrm{n}^{\circ} 4316$
} 
Remark that, for any integer $k \geq 2$,

$$
\tilde{P}_{k-1}=\left(\left(\begin{array}{c}
\hat{J}_{k-1} \\
0_{1 \times k}
\end{array}\right) \otimes \cdots \otimes\left(\begin{array}{c}
\hat{J}_{k-1} \\
0_{1 \times k}
\end{array}\right) \otimes I_{n}\right)^{T} \tilde{P}_{k}\left(\left(\begin{array}{c}
\hat{J}_{k-1} \\
0_{1 \times k}
\end{array}\right) \otimes \cdots \otimes\left(\begin{array}{c}
\hat{J}_{k-1} \\
0_{1 \times k}
\end{array}\right) \otimes I_{n}\right),
$$

where the chains of Kronecker products contain overall $m+1$ terms. Indeed, it is immediatly shown, with the help of (13), that

$$
\left(\left(\begin{array}{c}
\hat{J}_{k-1} \\
0_{1 \times k}
\end{array}\right) \otimes \cdots \otimes\left(\begin{array}{c}
\hat{J}_{k-1} \\
0_{1 \times k}
\end{array}\right) \otimes I_{n}\right)\left(v_{k}\left(z_{m}\right) \otimes \cdots \otimes v_{k}\left(z_{1}\right)\right)=\left(\begin{array}{c}
v_{k-1}\left(z_{m}\right) \\
0_{1 \times k}
\end{array}\right) \otimes \cdots \otimes\left(\begin{array}{c}
v_{k-1}\left(z_{1}\right) \\
0_{1 \times k}
\end{array}\right) .
$$

In other words, relation (25) expresses that the sum of the terms of degree at most $k-1$ in $\left(v_{k}\left(z_{m}\right) \otimes \cdots \otimes\right.$ $\left.v_{k}\left(z_{1}\right) \otimes I_{n}\right)^{*} \tilde{P}_{k}\left(v_{k}\left(z_{m}\right) \otimes \cdots \otimes v_{k}\left(z_{1}\right) \otimes I_{n}\right)$, is exactly equal to $\left(v_{k-1}\left(z_{m}\right) \otimes \cdots \otimes v_{k-1}\left(z_{1}\right) \otimes I_{n}\right)^{*} \tilde{P}_{k-1}\left(v_{k-1}\left(z_{m}\right) \otimes\right.$ $\left.\cdots \otimes v_{k-1}\left(z_{1}\right) \otimes I_{n}\right)$.

Remark also that, due to identity (23), the matrices $\tilde{P}_{k}$ are semidefinite positive.

\subsubsection{Proof of $(22 \mathrm{c}) \Rightarrow(22 \mathrm{~d})$}

Suppose there exists a solution to (21) analytic wrt $z_{i}, z_{i}^{*}$ in $\overline{\mathbb{D}}^{m}$. Then, by continuity and compactness (the convergence of the series being uniform in $\overline{\mathbb{D}}^{m}$ ), the polynomials obtained by truncating the terms with degree at least $k$ in one variable in the expansion (24), are solutions to inequality (21) in $\overline{\mathbb{D}}^{m}$, provided that $k$ is large enough.

\subsubsection{Proof of $(22 \mathrm{~d}) \Rightarrow(22 \mathrm{e})$.}

The form (22e) will be deduced from expansion (24). The following auxiliary result, which will be reused afterwards, permits to prove implication $(22 \mathrm{~d}) \Rightarrow(22 \mathrm{e})$ immediatly.

Lemma 6. Let $\tilde{P}_{k} \in \mathbb{C}^{k^{m} n \times k^{m} n}$ be a family of hermitian matrices such that (24) holds, in the sense of uniform convergence in $\overline{\mathbb{D}}^{m}$. Then,

(i) Without loss of generality, one may assume that, for any integer $k \geq 2$, identity (25) holds.

(ii) There exists a family of hermitian matrices $P_{k} \in \mathbb{C}^{k^{m} n \times k^{m} n}$ such that

$P\left(z_{1}, \ldots, z_{m}\right)=\lim _{k \rightarrow+\infty} \frac{1}{\left\|v_{k}\left(z_{1}\right)\right\|^{2} \cdots\left\|v_{k}\left(z_{m}\right)\right\|^{2}}\left(v_{k}\left(z_{m}\right) \otimes \cdots \otimes v_{k}\left(z_{1}\right) \otimes I_{n}\right)^{*} P_{k}\left(v_{k}\left(z_{m}\right) \otimes \cdots \otimes v_{k}\left(z_{1}\right) \otimes I_{n}\right)$,

uniformly in $\overline{\mathbb{D}}^{m}$.

(iii) Moreover, if

$$
\tilde{P}_{1}>0, \tilde{P}_{k} \geq 0, k \in \mathbb{N},
$$

then the coefficients $P_{k}$ in (26) may be supposed definite positive.

Proof. - Due to convergence property (24), one may suppose that the coefficients of each monomial in (24) converge when $k$ goes to infinity: one may as well replace directly the latter by their respective limit. When this operation is done, the terms of degree $k-2$ are already present, with the same coefficients, in

$$
\left(v_{k}\left(z_{m}\right) \otimes \cdots \otimes v_{k}\left(z_{1}\right) \otimes I_{n}\right)^{*} \tilde{P}_{k}\left(v_{k}\left(z_{m}\right) \otimes \cdots \otimes v_{k}\left(z_{1}\right) \otimes I_{n}\right) .
$$

This is what is expressed by (25), and this proves point (i).

- Consider now point (ii). The matrix $F_{k}$ being defined in (18), let

$$
P_{k} \stackrel{\text { def }}{=} \sum_{i_{1}, \ldots, i_{m}=0}^{k-1}\left(F_{k}^{i_{m}} \otimes \cdots \otimes F_{k}^{i_{1}} \otimes I_{n}\right) \tilde{P}_{k}\left(F_{k}^{i_{m}} \otimes \cdots \otimes F_{k}^{i_{1}} \otimes I_{n}\right)^{T}
$$


The matrix $P_{k}$ is clearly hermitian. One deduces from (20) that

$$
\begin{aligned}
& \left(v_{k}\left(z_{m}\right) \otimes \cdots \otimes v_{k}\left(z_{1}\right) \otimes I_{n}\right)^{*} P_{k}\left(v_{k}\left(z_{m}\right) \otimes \cdots \otimes v_{k}\left(z_{1}\right) \otimes I_{n}\right) \\
= & \sum_{i_{1}, \ldots, i_{m}=0}^{k-1}\left|z_{1}\right|^{2 i_{1}} \ldots\left|z_{m}\right|^{2 i_{m}}\left(\left(\begin{array}{c}
v_{k-i_{m}}\left(z_{m}\right) \\
0_{i_{1} \times 1}
\end{array}\right) \otimes \cdots \otimes\left(\begin{array}{c}
v_{k-i_{1}}\left(z_{1}\right) \\
0_{i_{m} \times 1}
\end{array}\right) \otimes I_{n}\right)^{*} \tilde{P}_{k}\left(\left(\begin{array}{c}
v_{k-i_{m}}\left(z_{m}\right) \\
0_{i_{m} \times 1}
\end{array}\right) \otimes \cdots \otimes\left(\begin{array}{c}
v_{k-i_{1}}\left(z_{1}\right) \\
0_{i_{1} \times 1}
\end{array}\right) \otimes \boldsymbol{I}_{n}\right) .
\end{aligned}
$$

As

$$
\sum_{i_{1}, \ldots, i_{m}=0}^{k-1}\left|z_{1}\right|^{2 i_{1}} \ldots\left|z_{m}\right|^{2 i_{m}}=\left\|v_{k}\left(z_{1}\right)\right\|^{2} \ldots\left\|v_{k}\left(z_{m}\right)\right\|^{2}
$$

we get

$$
\begin{aligned}
& \left\|v_{k}\left(z_{1}\right)\right\|^{2} \ldots\left\|v_{k}\left(z_{m}\right)\right\|^{2} P\left(z_{1}, \ldots, z_{m}\right)-\left(v_{k}\left(z_{m}\right) \otimes \cdots \otimes v_{k}\left(z_{1}\right) \otimes I_{n}\right)^{*} P_{k}\left(v_{k}\left(z_{m}\right) \otimes \cdots \otimes v_{k}\left(z_{1}\right) \otimes I_{n}\right) \\
& =\sum_{i_{1}, \ldots, i_{m}=0}^{k-1}\left|z_{1}\right|^{2 i_{1}} \ldots\left|z_{m}\right|^{2 i_{m}}\left[P\left(z_{1}, \ldots, z_{m}\right)\right. \\
& \left.-\left(\left(\begin{array}{c}
v_{k-i_{m}}\left(z_{m}\right) \\
0_{i_{m} \times 1}
\end{array}\right) \otimes \cdots \otimes\left(\begin{array}{c}
v_{k-i_{1}}\left(z_{1}\right) \\
0_{i_{1} \times 1}
\end{array}\right) \otimes I_{n}\right)^{*} \tilde{P}_{k}\left(\left(\begin{array}{c}
v_{k-i_{m}}\left(z_{m}\right) \\
0_{i_{m} \times 1}
\end{array}\right) \otimes \cdots \otimes\left(\begin{array}{c}
v_{k-i_{1}}\left(z_{1}\right) \\
0_{i_{1} \times 1}
\end{array}\right) \otimes I_{n}\right)\right] .
\end{aligned}
$$

Now, for any $\varepsilon>0$, there exists $k_{\varepsilon}$ such that, for any $k>k_{\varepsilon}$, for any $\left(z_{1}, \ldots, z_{m}\right) \in \overline{\mathbb{D}}^{m}$,

$$
\left\|P\left(z_{1}, \ldots, z_{m}\right)-\left(v_{k}\left(z_{m}\right) \otimes \cdots \otimes v_{k}\left(z_{1}\right) \otimes I_{n}\right)^{*} \tilde{P}_{k}\left(v_{k}\left(z_{m}\right) \otimes \cdots \otimes v_{k}\left(z_{1}\right) \otimes I_{n}\right)\right\|<\varepsilon .
$$

Distinguishing between the terms for which $\max \left\{i_{1}, \ldots, i_{m}\right\}<k-k_{\varepsilon}$ and $\max \left\{i_{1}, \ldots, i_{m}\right\} \geq k-k_{\varepsilon}$, permits to show that the norm of the expression in the left-hand side of (28) is bounded from above by

$$
\begin{gathered}
\varepsilon\left\|v_{k}\left(z_{1}\right)\right\|^{2} \ldots\left\|v_{k}\left(z_{m}\right)\right\|^{2}+2 c \sum_{\substack{k-1 \\
i_{1}, \ldots, i_{m}=0 \\
\max \left\{i_{1}, \ldots, i_{m}\right\} \geq k-k_{\varepsilon}}}\left|z_{1}\right|^{2 i_{1}} \ldots\left|z_{m}\right|^{2 i_{m}}
\end{gathered}
$$

where, due to property (24) and to (25), the constant

$$
c \stackrel{\text { def }}{=} \max _{i_{1}, \ldots, i_{m} \in \mathbb{N}} \max _{z_{i} \in \overline{\mathbb{D}}}\left\|\left(v_{i_{m}}\left(z_{m}\right) \otimes \cdots \otimes v_{i_{1}}\left(z_{1}\right) \otimes I_{n}\right)^{*} \tilde{P}_{k}\left(v_{i_{m}}\left(z_{m}\right) \otimes \cdots \otimes v_{i_{1}}\left(z_{1}\right) \otimes I_{n}\right)\right\|
$$

is finite and independent of $k, k_{\varepsilon}, \varepsilon, z_{1}, \ldots, z_{m}$. Now,

$$
\begin{aligned}
\sum_{\substack{i_{1}, \ldots, i_{m}=0 \\
\max \left\{i_{1}, \ldots, i_{m}\right\} \geq k-k_{\varepsilon}}}^{k-1}\left|z_{1}\right|^{2 i_{1}} \ldots\left|z_{m}\right|^{2 i_{m}}= & \sum_{i_{1}, \ldots, i_{m}=0}^{k-1}\left|z_{1}\right|^{2 i_{1}} \ldots\left|z_{m}\right|^{2 i_{m}}-\sum_{i_{1}, \ldots, i_{m}=0}^{k-k_{\varepsilon}-1}\left|z_{1}\right|^{2 i_{1}} \ldots\left|z_{m}\right|^{2 i_{m}} \\
= & \left\|v_{k}\left(z_{1}\right)\right\|^{2} \ldots\left\|v_{k}\left(z_{m}\right)\right\|^{2}-\left\|v_{k-k_{\varepsilon}}\left(z_{1}\right)\right\|^{2} \ldots\left\|v_{k-k_{\varepsilon}}\left(z_{m}\right)\right\|^{2} \\
= & \left(\left\|v_{k}\left(z_{1}\right)\right\|^{2}-\left\|v_{k-k_{\varepsilon}}\left(z_{1}\right)\right\|^{2}\right)\left\|v_{k}\left(z_{2}\right)\right\|^{2} \ldots\left\|v_{k}\left(z_{m}\right)\right\|^{2} \\
& +\cdots+\left\|v_{k-k_{\varepsilon}}\left(z_{1}\right)\right\|^{2} \ldots\left\|v_{k-k_{\varepsilon}}\left(z_{m-1}\right)\right\|^{2}\left(\left\|v_{k}\left(z_{m}\right)\right\|^{2}-\left\|v_{k-k_{\varepsilon}}\left(z_{m}\right)\right\|^{2}\right) .
\end{aligned}
$$

It turns out that, provided that $k>k_{\varepsilon}$,

$$
\begin{array}{r}
\left\|P\left(z_{1}, \ldots, z_{m}\right)-\frac{1}{\left\|v_{k}\left(z_{1}\right)\right\|^{2} \ldots\left\|v_{k}\left(z_{m}\right)\right\|^{2}}\left(v_{k}\left(z_{m}\right) \otimes \cdots \otimes v_{k}\left(z_{1}\right) \otimes I_{n}\right)^{*} P_{k}\left(v_{k}\left(z_{m}\right) \otimes \cdots \otimes v_{k}\left(z_{1}\right) \otimes I_{n}\right)\right\| \\
\leq \varepsilon+2 m c \sup _{r \in[0 ; 1)} r^{k-k_{\varepsilon}} \frac{1-r^{k_{\varepsilon}}}{1-r^{k}}
\end{array}
$$

uniformly in $\overline{\mathbb{D}}^{m}$. Notice that, for any fixed $k_{\varepsilon}$, the quantity

$$
\sup _{r \in[0 ; 1)} r^{k-k_{\varepsilon}} \frac{1-r^{k_{\varepsilon}}}{1-r^{k}}
$$

$\operatorname{RR} n^{\circ} 4316$ 
vanishes when $k$ goes to infinity. For large enough $k$, the latter quantity is less than $\varepsilon / 2 m c$ and, for any $\left(z_{1}, \ldots, z_{m}\right) \in \overline{\mathbb{D}}^{m}$

$$
\left\|P\left(z_{1}, \ldots, z_{m}\right)-\frac{1}{\left\|v_{k}\left(z_{1}\right)\right\|^{2} \ldots\left\|v_{k}\left(z_{m}\right)\right\|^{2}}\left(v_{k}\left(z_{m}\right) \otimes \cdots \otimes v_{k}\left(z_{1}\right) \otimes I_{n}\right)^{*} P_{k}\left(v_{k}\left(z_{m}\right) \otimes \cdots \otimes v_{k}\left(z_{1}\right) \otimes I_{n}\right)\right\|<2 \varepsilon .
$$

This proves the convergence property announced in (ii).

- Let us now prove (iii). Remark first that $P_{k} \geq 0$, due to (27). Let $u \in \mathbb{C}^{k^{m} n}$ such that $u^{*} P_{k} u=0$, it will be established that this implies $u=0$. In view of $(27)$, and due to the fact that $\tilde{P}_{k} \geq 0$, one sees that, for any $0 \leq i_{1}, \ldots, i_{m} \leq k-1$,

$$
u^{*}\left(F_{k}^{i_{m}} \otimes \cdots \otimes F_{k}^{i_{1}} \otimes I_{n}\right) \tilde{P}_{k}\left(F_{k}^{i_{m}} \otimes \cdots \otimes F_{k}^{i_{1}} \otimes I_{n}\right)^{T} u=0
$$

By the fact that

$$
F_{k}^{i T}=\left(\begin{array}{c}
I_{k-i} \\
0_{i \times(k-i)}
\end{array}\right)\left(0_{(k-i) \times i} \quad I_{k-i}\right)
$$

one deduces first, taking $i_{1}=\cdots=i_{m}=k-1$ and using convention (25), that

$$
\left\|\tilde{P}_{1}^{1 / 2}\left(\left(0_{1 \times(k-1)} 1\right) \otimes \cdots \otimes\left(0_{1 \times(k-1)} 1\right)\right) u\right\|^{2}=0,
$$

i.e., as $P_{1}>0$,

$$
\left(\left(0_{1 \times(k-1)} \quad 1\right) \otimes \cdots \otimes\left(0_{1 \times(k-1)} \quad 1\right)\right) u=0 .
$$

Take now $i_{1}=k-2, i_{2}=\cdots=i_{m}=k-1$. One gets:

$$
\left.\| \tilde{P}_{2}^{1 / 2}\left(\begin{array}{ll}
\left(0_{2 \times(k-2)}\right. & I_{2}
\end{array}\right) \otimes\left(\begin{array}{ll}
0_{1 \times(k-1)} & 1 \\
0_{1 \times(k-1)} & 0
\end{array}\right) \otimes \cdots \otimes\left(\begin{array}{cc}
0_{1 \times(k-1)} & 1 \\
0_{1 \times(k-1)} & 0
\end{array}\right)\right) u \|^{2}=0 .
$$

Remarking that

$$
\left(\begin{array}{ll}
0_{2 \times(k-2)} & I_{2}
\end{array}\right)=\left(\begin{array}{lll}
0_{1 \times(k-2)} & 1 & 0 \\
0_{1 \times(k-2)} & 0 & 1
\end{array}\right),
$$

and using (29) and convention (25), one now obtains

$$
\left\|\tilde{P}_{1}^{1 / 2}\left(\left(\begin{array}{lll}
0_{1 \times(k-2)} & 1 & 0
\end{array}\right) \otimes\left(0_{1 \times(k-1)} \quad 1\right) \otimes \cdots \otimes\left(0_{1 \times(k-1)} \quad 1\right)\right) u\right\|^{2}=0,
$$

so

$$
\left(\left(0_{1 \times(k-2)} \quad 1 \quad 0\right) \otimes\left(0_{1 \times(k-1)} \quad 1\right) \otimes \cdots \otimes\left(0_{1 \times(k-1)} \quad 1\right)\right) u=0 .
$$

One proves in this way that all the components of $u$ are null, provided that $u^{*} P_{k} u=0$. This shows that $P_{k}>0$ and achieves the proof of (iii), and of Lemma 6.

8.2.4 Proof of $(22 \mathrm{e}) \Rightarrow(22 \mathrm{f}) \Rightarrow(22 \mathrm{~g})$.

This part is the key of the demonstration. We shall mainly establish that $(22 \mathrm{f}) \Leftrightarrow(22 \mathrm{~g})$.

Kalman-Yakubovich-Popov lemma states the equivalence between positivity of a transfer and the existence of solution for a certain LMI (see Appendix). The following lemma, which will be used repeatedly, claims essentially that for transfers depending analytically upon parameters in a product of unitary disks, there exists solution of the LMI polynomial wrt the parameters and their conjugate. 
Lemma 7. Let $k, p, q \in \mathbb{N}$. Let $M\left(z_{2}, \ldots, z_{q}\right) \in \mathbb{C}^{(k+1) p \times(k+1) p}$ be hermitian, analytic wrt $z_{2}, \ldots, z_{q}, z_{2}^{*}, \ldots, z_{q}^{*}$ in $(\partial \mathbb{D})^{q-1}$. Properties (i) and (ii) below are equivalent.

(i) $\forall\left(z_{1}, \ldots, z_{q}\right) \in(\partial \mathbb{D})^{q}$,

$$
\left(v_{k+1}\left(z_{1}\right) \otimes I_{p}\right)^{*} M\left(z_{2}, \ldots, z_{q}\right)\left(v_{k+1}\left(z_{1}\right) \otimes I_{p}\right)<0 .
$$

(ii) $\exists \bar{k}_{1} \in \mathbb{N}, k_{1} \geq \bar{k}_{1} \Rightarrow \exists Q_{k_{1}, 1} \in \mathbb{C}^{k_{1}^{q-1} k p \times k_{1}^{q-1} k p}, Q_{k_{1}, 1}=Q_{k_{1}, 1}^{*}, \forall\left(z_{2}, \ldots, z_{q}\right) \in(\partial \mathbb{D})^{q-1}$,

$$
\begin{gathered}
M\left(z_{2}, \ldots, z_{q}\right)+\left(\begin{array}{c}
\hat{J}_{k} \otimes I_{p} \\
\tilde{J}_{k} \otimes I_{p}
\end{array}\right)^{T}\left(\begin{array}{cc}
Q\left(z_{2}, \ldots, z_{q}\right) & 0_{k p} \\
0_{k p} & -Q\left(z_{2}, \ldots, z_{q}\right)
\end{array}\right)\left(\begin{array}{l}
\hat{J}_{k} \otimes I_{p} \\
\tilde{J}_{k} \otimes I_{p}
\end{array}\right)<0, \\
Q\left(z_{2}, \ldots, z_{q}\right) \stackrel{\text { def }}{=} \frac{1}{\left\|v_{k_{1}}\left(z_{2}\right)\right\|^{2} \ldots\left\|v_{k_{1}}\left(z_{q}\right)\right\|^{2}}\left(v_{k_{1}}\left(z_{q}\right) \otimes \cdots \otimes v_{k_{1}}\left(z_{2}\right) \otimes I_{k p}\right)^{*} Q_{k_{1}, 1}\left(v_{k_{1}}\left(z_{q}\right) \otimes \cdots \otimes v_{k_{1}}\left(z_{2}\right) \otimes I_{k p}\right) .
\end{gathered}
$$

Proof. • 1ST STEP. Using (19), one has

$$
\left(v_{k+1}\left(z_{1}\right) \otimes I_{p}\right)=\left(\begin{array}{c}
I_{p} \\
z_{1}\left(I_{k p}-z_{1}\left(F_{k} \otimes I_{p}\right)\right)^{-1}\left(f_{k} \otimes I_{p}\right)
\end{array}\right) .
$$

Application of Kalman-Yakubovich-Popov lemma (reproduced in Appendix) then gets that property (i) is equivalent to: $\forall\left(z_{2}, \ldots, z_{q}\right) \in(\partial \mathbb{D})^{q-1}, \exists Q\left(z_{2}, \ldots, z_{q}\right) \in \mathbb{C}^{k p \times k p}, Q\left(z_{2}, \ldots, z_{q}\right)=Q\left(z_{2}, \ldots, z_{q}\right)^{*}$,

$$
M\left(z_{2}, \ldots, z_{q}\right)+\left(\begin{array}{c}
\hat{J}_{k} \otimes I_{p} \\
\tilde{J}_{k} \otimes I_{p}
\end{array}\right)^{T}\left(\begin{array}{cc}
Q\left(z_{2}, \ldots, z_{q}\right) & 0_{k p} \\
0_{k p} & -Q\left(z_{2}, \ldots, z_{q}\right)
\end{array}\right)\left(\begin{array}{c}
\hat{J}_{k} \otimes I_{p} \\
\check{J}_{k} \otimes I_{p}
\end{array}\right)<0 .
$$

- 2ND STEP. By compactness and continuity, the left-hand side of (30) may be bounded from above by $-\varepsilon I_{(k+1) p}, \varepsilon>0$, uniformly wrt $z_{2}, \ldots, z_{q}$ in $\partial \mathbb{D}$. Using evident notations for block decomposition of $M$, and notation (18), Schur complement permits to express equivalently (30) as the system of two inequalities (31), given by

$$
\left(f_{k} \otimes I_{p}\right)^{T} Q\left(f_{k} \otimes I_{p}\right)+M_{11}+\varepsilon I_{p}<0
$$

and

$$
\begin{aligned}
& \left(F_{k} \otimes I_{p}\right)^{T} Q\left(F_{k} \otimes I_{p}\right)-Q+M_{22}+\varepsilon I_{k p} \\
- & \left(\left(f_{k} \otimes I_{p}\right)^{T} Q\left(F_{k} \otimes I_{p}\right)+M_{12}\right)^{*}\left(\left(f_{k} \otimes I_{p}\right)^{T} Q\left(f_{k} \otimes I_{p}\right)+M_{11}+\varepsilon I_{p}\right)^{-1}\left(\left(f_{k} \otimes I_{p}\right)^{T} Q\left(F_{k} \otimes I_{p}\right)+M_{12}\right)<0 .
\end{aligned}
$$

Now, the existence of a matrix $Q$ fulfilling (31a) permits, at the cost of considering $Q+\eta I_{k p}$ instead of $Q$ for sufficiently large $\eta>0$, to assume that $M_{11}+\varepsilon I_{p}$ is negative for any $z_{2}, \ldots, z_{m}$ in $\partial \mathbb{D}$, and hence invertible. Applying [28, Theorem 13.1.1] with this change of variables, one gets that there exists a hermitian matrix $\tilde{Q}\left(z_{2}, \ldots, z_{m}\right)$ solution of the Riccati equation

$$
\begin{aligned}
& \left(F_{k} \otimes I_{p}\right)^{T} \tilde{Q}\left(F_{k} \otimes I_{p}\right)-\tilde{Q}+M_{22}+\varepsilon I_{k p} \\
- & \left(\left(f_{k} \otimes I_{p}\right)^{T} \tilde{Q}\left(F_{k} \otimes I_{p}\right)+M_{12}\right)^{*}\left(\left(f_{k} \otimes I_{p}\right)^{T} \tilde{Q}\left(f_{k} \otimes I_{p}\right)+M_{11}+\varepsilon I_{p}\right)^{-1}\left(\left(f_{k} \otimes I_{p}\right)^{T} \tilde{Q}\left(F_{k} \otimes I_{p}\right)+M_{12}\right)=0,
\end{aligned}
$$

and such that $\left(f_{k} \otimes I_{p}\right)^{T} \tilde{Q}\left(f_{k} \otimes I_{p}\right)+M_{11}+\varepsilon I_{p}<0$. This matrix is obviously solution of LMI (30), for any $z_{2}, \ldots, z_{m}$ in $\partial \mathbb{D}$.

Invoking again the results by Delchamps [9], extended by Kamen [26], the matrix $\tilde{Q}\left(z_{2}, \ldots, z_{q}\right)$ may be supposed analytic wrt $z_{2}, \ldots, z_{q}, z_{2}^{*}, \ldots, z_{q}^{*}$, and more simply polynomial, due to the strict inequality in (30). In other words, there exists $\overline{\bar{k}}_{1} \in \mathbb{N}$ such that: if $k_{1} \geq \overline{\bar{k}}_{1}$, then there exists a hermitian matrix $\tilde{Q}_{k_{1}, 1} \in \mathbb{C}^{k_{1}^{q-1} k p \times k_{1}^{q-1} k p}$ for which (30) is verified with $\tilde{Q}\left(z_{2}, \ldots, z_{q}\right)$ equal to

$$
\left(v_{k_{1}}\left(z_{q}\right) \otimes \cdots \otimes v_{k_{1}}\left(z_{2}\right) \otimes I_{k p}\right)^{*} \tilde{Q}_{k_{1}, 1}\left(v_{k_{1}}\left(z_{q}\right) \otimes \cdots \otimes v_{k_{1}}\left(z_{2}\right) \otimes I_{k p}\right) .
$$

$\operatorname{RR} n^{\circ} 4316$ 
- 3RD STEP. Using the same transformation than in Section 8.2.3 (Lemma 6, point (ii)), the family $\tilde{Q}_{k_{1}, 1}$ is transformed in a family $Q_{k_{1}, 1}$ of matrices of same size, such that, for the matrix defined in (32),

$$
\begin{aligned}
\lim _{k_{1} \rightarrow+\infty} \frac{1}{\left\|v_{k_{1}}\left(z_{2}\right)\right\|^{2} \cdots\left\|v_{k_{1}}\left(z_{q}\right)\right\|^{2}} & \left(v_{k_{1}}\left(z_{q}\right) \otimes \cdots \otimes v_{k_{1}}\left(z_{2}\right) \otimes I_{k p}\right)^{*} Q_{k_{1}, 1}\left(v_{k_{1}}\left(z_{q}\right) \otimes \cdots \otimes v_{k_{1}}\left(z_{2}\right) \otimes I_{k p}\right) \\
= & \left(v_{k_{1}}\left(z_{q}\right) \otimes \cdots \otimes v_{k_{1}}\left(z_{2}\right) \otimes I_{k p}\right)^{*} \tilde{Q}_{k_{1}, 1}\left(v_{k_{1}}\left(z_{q}\right) \otimes \cdots \otimes v_{k_{1}}\left(z_{2}\right) \otimes I_{k p}\right),
\end{aligned}
$$

with uniform convergence in $(\partial \mathbb{D})^{q-1}$. Thus, there exists $\bar{k}_{1} \in \mathbb{N}$, such that, for any $k_{1} \geq \bar{k}_{1}$, for any $\left(z_{2}, \ldots, z_{q}\right) \in(\partial \mathbb{D})^{q-1},(30)$ holds for $Q\left(z_{2}, \ldots, z_{q}\right)$ defined in (ii). This achieves the proof of Lemma 7 .

- Assume now that (22g) holds, that is: $\exists \bar{k}, \forall k \geq \bar{k}, \exists P_{k} \in \mathbb{C}^{k^{m} n \times k^{m} n}, P_{k}=P_{k}^{*}>0$, such that, $\forall\left(z_{1}, \ldots, z_{q}\right) \in$ $(\partial \mathbb{D})^{m},(21)$ is fulfilled with the hermitian definite positive matrix $P\left(z_{1}, \ldots, z_{q}\right) \in \mathbb{C}^{n \times n}$ defined by

$$
P\left(z_{1}, \ldots, z_{q}\right) \stackrel{\text { def }}{=} \frac{1}{\left\|v_{k}\left(z_{1}\right)\right\|^{2} \ldots\left\|v_{k}\left(z_{q}\right)\right\|^{2}}\left(v_{k}\left(z_{q}\right) \otimes \cdots \otimes v_{k}\left(z_{1}\right) \otimes I_{n}\right)^{*} P_{k}\left(v_{k}\left(z_{q}\right) \otimes \cdots \otimes v_{k}\left(z_{1}\right) \otimes I_{n}\right) .
$$

With the help of formulas (14) to (16), this may be written equivalently as: $\exists \bar{k}, \forall k \geq \bar{k}, \exists P_{k} \in \mathbb{C}^{k^{m} n \times k^{m} n}, P_{k}=$ $P_{k}^{*}>0, \forall\left(z_{1}, \ldots, z_{m}\right) \in(\partial \mathbb{D})^{m}$,

$\left(\begin{array}{c}v_{k}\left(z_{1}\right) \otimes I_{n} \\ z_{1}\left(v_{k}\left(z_{1}\right) \otimes I_{n}\right)\end{array}\right)^{*}\left(\begin{array}{cc}\left(I_{k} \otimes\left(A_{0}+z_{2} A_{2}+\cdots+z_{m} A_{m}\right)\right)^{*} P^{\prime}+P^{\prime}\left(I_{k} \otimes\left(A_{0}+z_{2} A_{2}+\cdots+z_{m} A_{m}\right)\right) & P^{\prime}\left(I_{k} \otimes A_{1}\right) \\ \left(I_{k} \otimes A_{1}\right)^{*} P^{\prime} & 0_{k n}\end{array}\right)\left(\begin{array}{c}v_{k}\left(z_{1}\right) \otimes I_{n} \\ z_{1}\left(v_{k}\left(z_{1}\right) \otimes I_{n}\right)\end{array}\right)<0$,

where by definition $P^{\prime} \in \mathbb{C}^{k n \times k n}$,

$$
P^{\prime}=P^{\prime}\left(z_{2}, \ldots, z_{m}\right) \stackrel{\text { def }}{=} \frac{1}{\left\|v_{k}\left(z_{2}\right)\right\|^{2} \cdots\left\|v_{k}\left(z_{m}\right)\right\|^{2}}\left(v_{k}\left(z_{m}\right) \otimes \cdots \otimes v_{k}\left(z_{2}\right) \otimes I_{k n}\right)^{*} P_{k}\left(v_{k}\left(z_{m}\right) \otimes \cdots \otimes v_{k}\left(z_{2}\right) \otimes I_{k n}\right) .
$$

One is now in position to eliminate the variable $z_{1}$. Taking into account identities (13) yields

$$
\left(\begin{array}{c}
v_{k}\left(z_{1}\right) \otimes I_{n} \\
z_{1} v_{k}\left(z_{1}\right) \otimes I_{n}
\end{array}\right)=\left(\begin{array}{c}
\hat{J}_{k} \otimes I_{n} \\
\check{J}_{k} \otimes I_{n}
\end{array}\right) v_{k+1}\left(z_{1}\right)
$$

Then, putting $p=n, q=m$ and

$$
M\left(z_{2}, \ldots, z_{m}\right) \stackrel{\text { def }}{=}\left(\begin{array}{c}
\hat{J}_{k} \otimes I_{n} \\
\tilde{J}_{k} \otimes I_{n}
\end{array}\right)^{T}\left(\begin{array}{cc}
\left(I_{k} \otimes\left(A_{0}+z_{2} A_{2}+\cdots+z_{m} A_{m}\right)\right)^{*} P^{\prime}+P^{\prime}\left(I_{k} \otimes\left(A_{0}+z_{2} A_{2}+\cdots+z_{m} A_{m}\right)\right) & P^{\prime}\left(I_{k} \otimes A_{1}\right) \\
\left(I_{k} \otimes A_{1}\right)^{*} P^{\prime} & 0_{k n}
\end{array}\right)\left(\begin{array}{c}
\hat{J}_{k} \otimes I_{n} \\
\tilde{J}_{k} \otimes I_{n}
\end{array}\right)
$$

in Lemma 7, provides equivalence between (22g) and the property: $\exists \bar{k}, \bar{k}_{1} \in \mathbb{N}, k_{1} \geq \bar{k}_{1}, k \geq \bar{k} \Rightarrow \exists P_{k} \in$ $\mathbb{C}^{k^{m} n \times k^{m} n}, P_{k}=P_{k}^{*}>0, \exists Q_{k_{1}, 1} \in \mathbb{C}^{k_{1}^{m-1} k n \times k_{1}^{m-1} k n}, Q_{k_{1}, 1}=Q_{k_{1}, 1}^{*}, \forall\left(z_{2}, \ldots, z_{m}\right) \in(\partial \mathbb{D})^{m-1}$,

$$
\begin{aligned}
& \left(\begin{array}{c}
\hat{J}_{k} \otimes I_{n} \\
\tilde{J}_{k} \otimes I_{n}
\end{array}\right)^{T}\left[\left(\begin{array}{cc}
\left(I_{k} \otimes\left(A_{0}+z_{2} A_{2}+\cdots+z_{m} A_{m}\right)\right)^{*} P^{\prime}+P^{\prime}\left(I_{k} \otimes\left(A_{0}+z_{2} A_{2}+\cdots+z_{m} A_{m}\right)\right) & P^{\prime}\left(I_{k} \otimes A_{1}\right) \\
\left(I_{k} \otimes A_{1}\right)^{*} P^{\prime} & 0_{k n}
\end{array}\right)\right. \\
& \left.+\left(\begin{array}{cc}
Q_{1}^{\prime} & 0_{k n} \\
0_{k n} & -Q_{1}^{\prime}
\end{array}\right)\right]\left(\begin{array}{c}
\hat{J}_{k} \otimes I_{n} \\
\tilde{J}_{k} \otimes I_{n}
\end{array}\right)<0
\end{aligned}
$$

where $P^{\prime}$ is defined in (33) and $Q_{1}^{\prime} \in \mathbb{C}^{k n \times k n}$ is defined by

$$
Q_{1}^{\prime}=Q_{1}^{\prime}\left(z_{2}, \ldots, z_{m}\right) \stackrel{\text { def }}{=} \frac{1}{\left\|v_{k_{1}}\left(z_{2}\right)\right\|^{2} \ldots\left\|v_{k_{1}}\left(z_{m}\right)\right\|^{2}}\left(v_{k_{1}}\left(z_{m}\right) \otimes \cdots \otimes v_{k_{1}}\left(z_{2}\right) \otimes I_{k n}\right)^{*} Q_{k_{1}, 1}\left(v_{k_{1}}\left(z_{m}\right) \otimes \cdots \otimes v_{k_{1}}\left(z_{2}\right) \otimes I_{k n}\right) .
$$

Remark that, without loss of generality, one may as well suppose that $\bar{k}=\bar{k}_{1}, k=k_{1}$ in the previous formula,

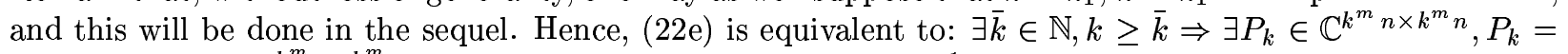
$P_{k}^{*}>0, \exists Q_{k, 1} \in \mathbb{C}^{k^{m} n \times k^{m} n}, Q_{k, 1}=Q_{k, 1}^{*}, \forall\left(z_{2}, \ldots, z_{m}\right) \in(\partial \mathbb{D})^{m-1}$,

$$
\begin{array}{r}
\left(J_{k}^{1} \otimes I_{n}\right)^{T}\left(\begin{array}{cc}
\left(I_{k} \otimes\left(A_{0}+z_{2} A_{2}+\cdots+z_{m} A_{m}\right)\right)^{*} P^{\prime}+P^{\prime}\left(I_{k} \otimes\left(A_{0}+z_{2} A_{2}+\cdots+z_{m} A_{m}\right)\right) & P^{\prime}\left(I_{k} \otimes A_{1}\right) \\
& P^{\prime}\left(I_{k} \otimes A_{1}\right) \\
+ & 0_{k n}
\end{array}\right)\left(J_{k}^{1} \otimes I_{n}\right) \\
+\left(\hat{J}_{k, 1}^{1} \otimes I_{n}\right)^{T} Q_{1}^{\prime}\left(\hat{J}_{k, i}^{1} \otimes I_{n}\right)-\left(\check{J}_{k, 1}^{1} \otimes I_{n}\right)^{T} Q_{1}^{\prime}\left(\check{J}_{k, 1}^{1} \otimes I_{n}\right)<0, \quad(34)
\end{array}
$$


where $P^{\prime}\left(z_{2}, \ldots, z_{m}\right), Q_{1}^{\prime}\left(z_{2}, \ldots, z_{m}\right)$ have been defined previously.

- The same mechanism will now be applied by induction.

Let $0 \leq i \leq m-1$. Suppose that (22g) is equivalent to: $\exists \bar{k} \in \mathbb{N}, k \geq \bar{k} \Rightarrow \exists P_{k} \in \mathbb{C}^{k^{m} n \times k^{m} n}, P_{k}=$ $P_{k}^{*}>0, \exists Q_{k, 1} \in \mathbb{C}^{k^{m} n \times k^{m} n}, Q_{k, 1}=Q_{k, 1}^{*}, \ldots, \exists Q_{k, i} \in \mathbb{C}^{k^{m-i+1}(k+1)^{i-1} n \times k^{m-i+1}(k+1)^{i-1} n}, Q_{k, i}=Q_{k, i}^{*}$, $\forall\left(z_{i+1}, \ldots, z_{m}\right) \in(\partial \mathbb{D})^{m-i}$

$$
R_{k, i}^{\mathbb{C}}\left(P^{(i)}\left(z_{i+1}, \ldots, z_{m}\right), Q_{1}^{(i)}\left(z_{i+1}, \ldots, z_{m}\right), \ldots, Q_{i}^{(i)}\left(z_{i+1}, \ldots, z_{m}\right), z_{i+1}, \ldots, z_{m}\right)<0 .
$$

In (35), we have set up, for any $0 \leq i \leq m-1$, the matrix $P^{(i)}\left(z_{i+1}, \ldots, z_{m}\right)$ as

$$
P^{(i)}\left(z_{i+1}, \ldots, z_{m}\right) \stackrel{\text { def }}{=} \frac{1}{\left\|v_{k}\left(z_{i+1}\right)\right\|^{2} \ldots\left\|v_{k}\left(z_{m}\right)\right\|^{2}}\left(v_{k}\left(z_{m}\right) \otimes \cdots \otimes v_{k}\left(z_{i+1}\right) \otimes I_{k n}\right)^{*} P_{k}\left(v_{k}\left(z_{m}\right) \otimes \cdots \otimes v_{k}\left(z_{i+1}\right) \otimes I_{k n}\right),
$$

and similarly for the matrices $Q_{1}^{(i)}\left(z_{i+1}, \ldots, z_{m}\right), \ldots, Q_{i}^{(i)}\left(z_{i+1}, \ldots, z_{m}\right)$. The size of the previous matrix functions is given by:

$$
P^{(i)} \in \mathbb{C}^{k^{i} n \times k^{i} n}, \quad Q_{i^{\prime}}^{(i)} \in \mathbb{C}^{k^{i-i^{\prime}+1}(k+1)^{i^{\prime}-1} n \times k^{i-i^{\prime}+1}(k+1)^{i^{\prime}-1} n}, 1 \leq i^{\prime} \leq i .
$$

The value of $R_{k, i}^{\mathbb{C}}\left(P^{(i)}\left(z_{i+1}, \ldots, z_{m}\right), Q_{1}^{(i)}\left(z_{i+1}, \ldots, z_{m}\right), \ldots, Q_{i}^{(i)}\left(z_{i+1}, \ldots, z_{m}\right), z_{i+1}, \ldots, z_{m}\right)$ in (35) is a complicated expression, sum of $i+1$ terms. The first term is the result of the right- and left-product of

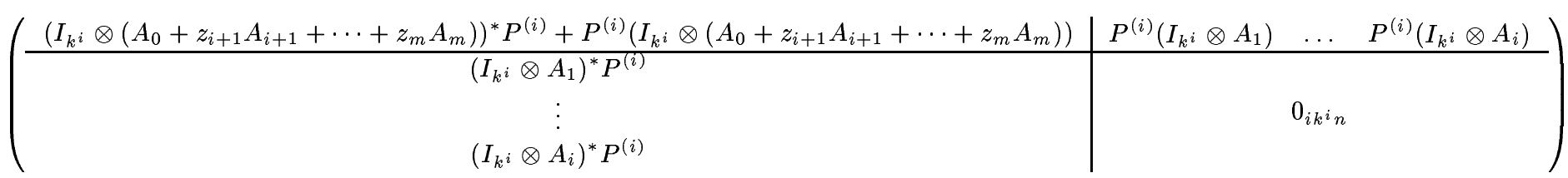

by $\left(J_{k}^{i} \otimes I_{n}\right)$ and its conjugate. The sum of the remaining $i$ terms in $R_{k, i}^{\mathbb{C}}$ is equal to

$$
\sum_{i^{\prime}=1}^{i}\left(\left(\hat{J}_{k, i^{\prime}}^{i} \otimes I_{n}\right)^{T} Q_{i^{\prime}}^{(i)}\left(\hat{J}_{k, i^{\prime}}^{i} \otimes I_{n}\right)-\left(\check{J}_{k, i^{\prime}}^{i} \otimes I_{n}\right)^{T} Q_{i^{\prime}}^{(i)}\left(\check{J}_{k, i^{\prime}}^{i} \otimes I_{n}\right)\right)
$$

The matrix $R_{k, i}^{\mathbb{C}}$ is an element of $\mathbb{C}^{(k+1)^{i} n \times(k+1)^{i} n}$. Notice that for $i=1, R_{k, 1}^{\mathbb{C}}$ is exactly the expression in the left-hand side of (34), while for $i=m, R_{k, m}^{\mathbb{C}}$ is just $R_{k}^{\mathbb{C}}$ given in (5).

Now that the meaning of (35) is clear, we shall eliminate the variable $z_{i+1}$ and establish that (35) holds for $i+1$. This operation has been achieved previously for the case $i=0$.

The following functional identity is easily shown:

$$
P^{(i)}=\frac{1}{\left\|v_{k}\left(z_{i+1}\right)\right\|^{2}}\left(v_{k}\left(z_{i+1}\right) \otimes I_{k^{i} n}\right)^{*} P^{(i+1)}\left(v_{k}\left(z_{i+1}\right) \otimes I_{k^{i} n}\right),
$$

and similarly

$$
Q_{i^{\prime}}^{(i)}=\frac{1}{\left\|v_{k}\left(z_{i+1}\right)\right\|^{2}}\left(v_{k}\left(z_{i+1}\right) \otimes I_{k^{i-i^{\prime}+1}(k+1)^{i^{\prime}-1} n}\right)^{*} Q_{i^{\prime}}^{(i+1)}\left(v_{k}\left(z_{i+1}\right) \otimes I_{k^{i-i^{\prime}+1}(k+1)^{i^{\prime}-1} n}\right), 1 \leq i^{\prime} \leq i .
$$

Using (14) to establish that

$$
\begin{aligned}
P^{(i)}\left(I_{k^{i}} \otimes A_{i}\right) & =\frac{1}{\left\|v_{k}\left(z_{i+1}\right)\right\|^{2}}\left(v_{k}\left(z_{i+1}\right) \otimes I_{k^{i} n}\right)^{*} P^{(i+1)}\left(v_{k}\left(z_{i+1}\right) \otimes I_{k^{i} n}\right)\left(I_{k^{i}} \otimes A_{i}\right) \\
& =\frac{1}{\left\|v_{k}\left(z_{i+1}\right)\right\|^{2}}\left(v_{k}\left(z_{i+1}\right) \otimes I_{k^{i} n}\right)^{*} P^{(i+1)}\left(I_{k^{i+1}} \otimes A_{i}\right)\left(v_{k}\left(z_{i+1}\right) \otimes I_{k^{i} n}\right),
\end{aligned}
$$

and then the three properties enunciated in Lemma 5, the product of the left-hand side of (35) by the (positive) expression $\left\|v_{k}\left(z_{i+1}\right)\right\|^{2}$ is recognized as

$$
\left(v_{k+1}\left(z_{i+1}\right) \otimes I_{k^{i} n}\right)^{*} R_{k, i+1}^{\mathbb{C}}\left(P^{(i+1)}\left(z_{i+2}, \ldots, z_{m}\right), Q_{1}^{(i+1)}\left(z_{i+2}, \ldots, z_{m}\right), \ldots, Q_{i}^{(i+1)}\left(z_{i+2}, \ldots, z_{m}\right), 0_{k(k+1)^{i} n}, z_{i+2}, \ldots, z_{m}\right)\left(v_{k+1}\left(z_{i+1}\right) \otimes I_{k^{i} n}\right) .
$$


It is now possible to apply Lemma 7 as previously, with $p=k^{i} n, q=m-i$, and

$M\left(z_{i+2}, \ldots, z_{m}\right) \stackrel{\text { def }}{=} R_{k, i+1}^{\mathbb{C}}\left(P^{(i+1)}\left(z_{i+2}, \ldots, z_{m}\right), Q_{1}^{(i+1)}\left(z_{i+2}, \ldots, z_{m}\right), \ldots, Q_{i}^{(i+1)}\left(z_{i+2}, \ldots, z_{m}\right), 0_{k(k+1)^{i} n}, z_{i+2}, \ldots, z_{m}\right)$.

This yields the equivalence between (22g) and: $\exists \bar{k} \in \mathbb{N}, k \geq \bar{k} \Rightarrow \exists P_{k} \in \mathbb{C}^{k^{m} n \times k^{m} n}, P_{k}=P_{k}^{*}>0, \exists Q_{k, 1} \in$ $\mathbb{C}^{k^{m} n \times k^{m} n}, Q_{k, 1}=Q_{k, 1}^{*}, \ldots, \exists Q_{k, i+1} \in \mathbb{C}^{k^{m-i}(k+1)^{i} n \times k^{m-i}(k+1)^{i} n}, Q_{k, i+1}=Q_{k, i+1}^{*}, \forall\left(z_{i+2}, \ldots, z_{m}\right) \in$ $(\partial \mathbb{D})^{m-i-1}$,

$R_{k, i+1}^{\mathbb{C}}\left(P^{(i+1)}\left(z_{i+2}, \ldots, z_{m}\right), Q_{1}^{(i+1)}\left(z_{i+2}, \ldots, z_{m}\right), \ldots, Q_{i}^{(i+1)}\left(z_{i+2}, \ldots, z_{m}\right), Q_{i+1}^{(i+1)}\left(z_{i+2}, \ldots, z_{m}\right), z_{i+2}, \ldots, z_{m}\right)<0$.

Property (35) is hence true for rank $i+1$ and, by recursion, for rank $m$. For this latter value, one ends up precisely in (22f).

This achieves the demonstration of the equivalence $(22 \mathrm{f}) \Leftrightarrow(22 \mathrm{~g})$. Thus, $(22 \mathrm{e}) \Rightarrow(22 \mathrm{~g}) \Rightarrow(22 \mathrm{f}) \Rightarrow(22 \mathrm{~g})$, and, as a conclusion, $(22 \mathrm{e}) \Rightarrow(22 \mathrm{f}) \Rightarrow(22 \mathrm{~g})$, as announced.

\subsubsection{Proof of $(22 \mathrm{~h}) \Rightarrow(22 \mathrm{a})$.}

This implication is proved by subharmonicity and continuity argument. Using the fact that the map $\overline{\mathbb{C}^{+}} \cup$ $\{\infty\} \rightarrow \overline{\mathbb{D}}, s_{z} \mapsto\left(1-s_{z}\right) /\left(1+s_{z}\right)$ is one-to-one, one proves [5]

$$
\begin{aligned}
\max _{z_{i} \in \overline{\mathbb{D}}} \rho\left(e^{A_{0}+z_{1} A_{1}+\cdots+z_{m} A_{m}}\right) & =\sup _{s_{i} \in \overline{\mathbb{C}^{+}}} \rho\left(e^{A_{0}+\left(1-s_{1}\right) /\left(1+s_{1}\right) A_{1}+\cdots+\left(1-s_{m}\right) /\left(1+s_{m}\right) A_{m}}\right) \\
& =\sup _{s_{i} \in j \mathbb{R}} \rho\left(e^{A_{0}+\left(1-s_{1}\right) /\left(1+s_{1}\right) A_{1}+\cdots+\left(1-s_{m}\right) /\left(1+s_{m}\right) A_{m}}\right)=\max _{z_{i} \in \partial \mathbb{D}} \rho\left(e^{A_{0}+z_{1} A_{1}+\cdots+z_{m} A_{m}}\right) .
\end{aligned}
$$

The desired property ensues from the fact that (22a) holds, if and only if the previous expression is smaller than 1.

\subsection{Proof of Theorem 3}

The key idea is to introduce for each $r_{i} \in[-1 ;+1]$ the change of variable $r_{i}=\left(z_{i}+z_{i}^{*}\right) / 2, z_{i} \in \overline{\mathbb{D}}$, and to achieve slight adaptations of the proof of Theorem 1 . Consider in consequence the parameter-dependent Lyapunov inequality

$$
\begin{aligned}
P\left(z_{1}, \ldots, z_{m}\right)\left(A_{0}+\frac{z_{1}+z_{1}^{*}}{2} A_{1}+\right. & \left.+\frac{z_{m}+z_{m}^{*}}{2} A_{m}\right) \\
& +\left(A_{0}+\frac{z_{1}+z_{1}^{*}}{2} A_{1}+\cdots+\frac{z_{m}+z_{m}^{*}}{2} A_{m}\right)^{*} P\left(z_{1}, \ldots, z_{m}\right)<0 .
\end{aligned}
$$

The proof proceeds along the following steps.

$$
\begin{aligned}
& \forall\left(z_{1}, \ldots, z_{m}\right) \in \overline{\mathbb{D}}^{m}, A_{0}+\frac{z_{1}+z_{1}^{*}}{2} A_{1}+\cdots+\frac{z_{m}+z_{m}^{*}}{2} A_{m} \text { is Hurwitz, } \\
& \Rightarrow \forall\left(z_{1}, \ldots, z_{m}\right) \in \overline{\mathbb{D}}^{m}, \exists P\left(z_{1}, \ldots, z_{m}\right)=P\left(z_{1}, \ldots, z_{m}\right)^{*}>0 \text { fulfilling (36), } \\
& \Rightarrow \quad \exists P\left(z_{1}, \ldots, z_{m}\right)=P\left(z_{1}, \ldots, z_{m}\right)^{*}>0 \text { analytic wrt } z_{1}, \ldots, z_{m}, z_{1}^{*}, \ldots, z_{m}^{*} \text { in } \overline{\mathbb{D}}^{m} \text {, fulfilling (36), } \\
& \Rightarrow \quad \exists \bar{k} \in \mathbb{N}, \forall k \geq \bar{k}, \exists P\left(z_{1}, \ldots, z_{m}\right)=P\left(z_{1}, \ldots, z_{m}\right)^{*}>0 \text { polynomial of degree } k-1 \text { wrt } z_{1}, \ldots, z_{m} \text {, } \\
& z_{1}^{*}, \ldots, z_{m}^{*} \text { in } \overline{\mathbb{D}}^{m} \text {, fulfilling (36), } \\
& \Rightarrow \quad \exists \bar{k} \in \mathbb{N}, \forall k \geq \bar{k}, \exists P_{k} \in \mathbb{C}^{k^{m} n \times k^{m} n}, P_{k}=P_{k}^{*}>0, \forall\left(z_{1}, \ldots, z_{m}\right) \in \overline{\mathbb{D}}^{m}, P\left(z_{1}, \ldots, z_{m}\right) \text { fulfills (36), with } \\
& P\left(z_{1}, \ldots, z_{m}\right) \stackrel{\text { def }}{=} \frac{1}{\left\|v_{k}\left(z_{1}\right)\right\|^{2} \ldots\left\|v_{k}\left(z_{m}\right)\right\|^{2}}\left(v_{k}\left(z_{1}\right) \otimes \cdots \otimes v_{k}\left(z_{m}\right) \otimes I_{n}\right)^{*} P_{k}\left(v_{k}\left(z_{1}\right) \otimes \cdots \otimes v_{k}\left(z_{m}\right) \otimes I_{n}\right) \\
& \Rightarrow \exists \bar{k} \in \mathbb{N}, \forall k \geq \bar{k}, \mathrm{LMI}_{k}^{\mathbb{R}} \text { is solvable (same } \bar{k} \text { and } P_{k} \text { than in (37e)), } \\
& \Rightarrow \quad \exists \bar{k} \in \mathbb{N}, \forall k \geq \bar{k}, \exists P_{k} \in \mathbb{C}^{k^{m} n \times k^{m} n}, P_{k}=P_{k}^{*}>0 \text { (same } \bar{k} \text { and } P_{k} \text { than in (37e)), such that, } \\
& \forall\left(z_{1}, \ldots, z_{m}\right) \in(\partial \mathbb{D})^{m}, P(z) \text { as in (37e) fulfills }(36) \\
& \Rightarrow \quad \forall\left(z_{1}, \ldots, z_{m}\right) \in(\partial \mathbb{D})^{m}, \exists P\left(z_{1}, \ldots, z_{m}\right)=P\left(z_{1}, \ldots, z_{m}\right)^{*}>0 \text { fulfilling (36), } \\
& \Rightarrow \text { (37a). }
\end{aligned}
$$


Here, the implications $(37 \mathrm{~g}) \Rightarrow(37 \mathrm{~h}) \Rightarrow(37 \mathrm{a}) \Rightarrow(37 \mathrm{~b})$ are evident. The other implications are deduced as in Section 8.2 for the proof of Theorem 1 . The only difference lies in the proof of $(37 \mathrm{e}) \Rightarrow(37 \mathrm{f}) \Rightarrow(37 \mathrm{~g})$, instead of $(22 \mathrm{e}) \Rightarrow(22 \mathrm{f}) \Rightarrow(22 \mathrm{~g})$ (see Section 8.2 .4$)$. For $P$ as in $(37 \mathrm{e})$, the quantity

$$
P\left(z_{1}, \ldots, z_{m}\right)\left(2 A_{0}+\left(z_{1}+z_{1}^{*}\right) A_{1}+\cdots+\left(z_{m}+z_{m}^{*}\right) A_{m}\right)+\left(2 A_{0}+\left(z_{1}+z_{1}^{*}\right) A_{1}+\cdots+\left(z_{m}+z_{m}^{*}\right) A_{m}\right)^{*} P\left(z_{1}, \ldots, z_{m}\right)
$$

is equal to the right- and left-product of the matrix

$$
\left(\begin{array}{cc}
\left(I_{k} \otimes\left(2 A_{0}+\left(z_{2}+z_{2}^{*}\right) A_{2}+\cdots+\left(z_{m}+z_{m}^{*}\right) A_{m}\right)\right)^{*} P^{\prime}+P^{\prime}\left(I_{k} \otimes\left(A_{0}+\left(z_{2}+z_{2}^{*}\right) A_{2}+\cdots+\left(z_{m}+z_{m}^{*}\right) A_{m}\right)\right) & P^{\prime}\left(I_{k} \otimes A_{1}\right)+\left(I_{k} \otimes A_{1}\right)^{*} P^{\prime} \\
P^{\prime}\left(I_{k} \otimes A_{1}\right)+\left(I_{k} \otimes A_{1}\right)^{*} P^{\prime} & 0_{k n}
\end{array}\right)
$$

(with $P^{\prime}$ defined as in (33)) by

$$
\left(\begin{array}{c}
v_{k}\left(z_{1}\right) \otimes I_{n} \\
z_{1}\left(v_{k}\left(z_{1}\right) \otimes I_{n}\right)
\end{array}\right)
$$

This is the origin of the differences between the two results.

\section{Conclusion}

Robust stability of linear systems with several scalar (complex or real) parameters has been studied. For each problem, a family of simple LMIs, indexed by a positive integer $k$, is provided. Their solvability is sufficient for robust stability, and the corresponding conditions are less and less conservative when $k$ increases. Conversely, the criteria presented are also necessary, in the sense that: if robust stability holds, then the corresponding LMI problems are solvable from a certain $k$ and beyond. Roughly speaking, this integer $k$ is linked to the degree of parameter-dependent Lyapunov function, polynomial wrt the parameters and their conjugate.

The issue of robust stability may be easily expressed by conditions involving structured singular values, the central tool of $\mu$-analysis. Their evaluation is known to be a NP-hard problem, for which no convex algorithm is supposed to exist (except if $\mathrm{P}=\mathrm{NP} \ldots$ ). The results presented here furnish a way of approximating this nonconvex problem, by a sequence of convex ones (namely LMIs), of increasing size. The accuracy of the approximation is only limited by computation time, and this is, to our knowledge, an original characteristic of this approach. Furthermore, it has to be stressed that the method of obtention of the LMIs is constructive, and the latter are constructed by limited set of elementary algebraic matrix operations. In consequence, the derived algorithms are immediatly implementable in a MATLAB/ SCILAB-like environment.

Further research includes the following aspects.

1. Determination of the degree of accuracy needed to test the robust stability of any specific system; that is of an a priori (upper) estimate on the least $k$, if any, for which the LMIs are solvable. More generally, the complexity issues have to be analyzed.

2. Extension of the results to robust $H_{\infty}$ and $H_{2}$ performance evaluation, for systems with scalar parameters (robust $H_{\infty}$ performance is solved in [3] for systems with one complex parameter). Extension to the case of polynomial or LFT dependency wrt the parameters (see $[2,4]$ for the single complex parameter case).

3. Extension to the evaluation of general structured singular values with scalar repeated blocks.

\section{A Appendix - Discrete-time version of Kalman-Yakubovich-Popov lemma}

Initially appeared in [43], the result has been first published under its discrete-time form by Szegö and Kalman [35]. We use the statement as expressed e.g. in [33]. A proof of the result in the complex case may be found in [29, Theorem 1.11.1 and Remark 1.11.1].

Let $A \in \mathbb{C}^{n \times n}, B \in \mathbb{C}^{n \times p}, M=M^{*} \in \mathbb{C}^{(p+n) \times(p+n)}$.

RR $n^{\circ} 4316$ 
Lemma A. If $\operatorname{det}\left(I_{n}-z A\right) \neq 0$ for any $z \in \partial \mathbb{D}$, then the following two statements are equivalent.

(i) There exists $Q=Q^{*} \in \mathbb{C}^{n \times n}$ such that

$$
0>\left(\begin{array}{cc}
B^{*} Q B & B^{*} Q A \\
A^{*} Q B & A^{*} Q A-Q
\end{array}\right)+M=\left(\begin{array}{cc}
B & A \\
0_{n \times p} & I_{p}
\end{array}\right)^{*}\left(\begin{array}{cc}
Q & 0_{n} \\
0_{n} & -Q
\end{array}\right)\left(\begin{array}{cc}
B & A \\
0_{n \times p} & I_{p}
\end{array}\right)+M .
$$

(ii) For any $z \in \partial \mathbb{D}$,

$$
\left(\begin{array}{c}
I_{n} \\
z\left(I_{n}-z A\right)^{-1} B
\end{array}\right)^{*} M\left(\begin{array}{c}
I_{n} \\
z\left(I_{n}-z A\right)^{-1} B
\end{array}\right)<0 .
$$

When in the statements the matrices $A, B, M$ are real, then $Q$ is real, symmetric.

\section{References}

[1] P. Apkarian, H.D. Tuan (2000). Parameterized LMIs in control theory, SIAM Journal on Control and Optimization 38 no 4, 1241-1264

[2] P.-A. Bliman (to appear). Lyapunov equation for the stability of linear delay systems of retarded and neutral type, IEEE Trans. Automat. Control

[3] P.-A. Bliman (2001). Nonconservative LMI criteria for characterization of delay-independent properties of delay systems. Application to stability and input-output analysis of systems with complex parameter, Report research no 4278, INRIA, http: //www.inria.fr/rrrt/rr-4278.html

[4] P.-A. Bliman (2001). LMI approach to spectral stabilizability of linear delay systems and stabilizability of linear systems with complex parameter, Proc. of 40 th IEEE CDC, Orlando (Florida)

[5] S. Boyd, C.A. Desoer (1985). Subharmonic functions and performance bounds on linear time-invariant feedback systems, IMA Journal of Mathematical Control \& Information 2, 153-170

[6] S. Boyd, L. El Ghaoui, E. Feron, V. Balakrishnan (1994). Linear matrix inequalities in system and control theory, SIAM Studies in Applied Mathematics vol. 15

[7] R.P. Braatz, P.M. Young, J.C. Doyle, M. Morari (1994). Computational complexity of $\mu$ calculation, IEEE Trans. Automat. Control 39 no 5, 1000-1002

[8] J. Chen, H.A. Latchman (1995). Frequency sweeping tests for stability independent of delay, IEEE Trans. Automat. Control 40 no 9, 1640-1645

[9] D.F. Delchamps (1984). Analytic feedback control and the algebraic Riccati equation, IEEE Trans. Automat. Control AC-29 no 11, 1031-1033

[10] M. Dettori, C.W. Scherer (1998). Robust stability analysis for parameter dependent systems using full block S-procedure, Proc. of 37th IEEE CDC, Tampa (Florida), 2798-2799

[11] M. Dettori, C.W. Scherer (2000). New robust stability and performance conditions based on parameter dependent multipliers, Proc. of 39th IEEE CDC, Sydney (Australia)

[12] J.C. Doyle (1982). Analysis of feedback systems with structured uncertainties, IEE Proc. Part D 129 no $6,242-250$

[13] E. Feron, P. Apkarian, P. Gahinet (1996). Analysis and synthesis of robust control systems via parameter-dependent Lyapunov functions, IEEE Trans. Automat. Control 41 no 7, 1041-1046 
[14] M. Fu (1997). The real structured singular value is hardly approximable, IEEE Trans. Automat. Control 42 no $9,1286-1288$

[15] P. Gahinet, P. Apkarian, M. Chilali (1996). Affine parameter-dependent Lyapunov functions and real parametric uncertainty, IEEE Trans. Automat. Control 41 no 3, 436-442

[16] J.C. Geromel, M.C. de Oliveira, L. Hsu (1998). LMI characterization of structural and robust stability, Linear Algebra Appl. 285 no 1-3, 69-80

[17] J.K. Hale (1977). Theory of functional differential equations, Applied Mathematical Sciences 3, Springer Verlag, New York

[18] J.K. Hale, E.F. Infante, F.S.P. Tsen (1985). Stability in linear delay equations, J. Math. Anal. Appl. 115, $533-555$

[19] D. Hertz, E.I. Jury, E. Zeheb (1984). Stability independent and dependent of delay for delay differential systems, J. Franklin Institute $\mathbf{3 1 8}$ no 3, 143-150

[20] T. Iwasaki, S. Hara (1998). Well-posedness of feedback systems: insights into exact robustness analysis and approximate computations, IEEE Trans. Automat. Control 43 no 5, 619-630

[21] T. Iwasaki (1998). LPV system analysis with quadratic separator, Proc. of 37th IEEE CDC, Tampa (Florida)

[22] E.W. Kamen (1980). On the relationship between zero criteria for two-variable polynomials and asymptotic stability of delay differential equations, IEEE Trans. Automat. Control 25 no 5, 983-984

[23] E.W. Kamen (1982). Linear systems with commensurate time delays: stability and stabilization independent of delay, IEEE Trans. Automat. Control 27 no 2, 367-375

[24] E.W. Kamen (1983). Correction to "Linear systems with commensurate time delays: stability and stabilization independent of delay", IEEE Trans. Automat. Control 28 no 2, 248-249

[25] E.W. Kamen (1984). Pointwise stability and feedback control of linear systems with noncommensurate time delays, Acta Applicandae Mathematica 2, 159-184

[26] E.W. Kamen (1985). Stabilization of linear spatially-distributed continuous-time and discrete-time systems, Multidimensional systems theory. Progress, directions and open problems in multidimensional systems, Mathematics and its Applications 16, D. Reidel Publishing Co, Dordrecht, N.K. Bose Ed., $101-146$

[27] N.N. Krasovskii (1963). Stability of motion, Stanford University Press

[28] P. Lancaster, L. Rodman (1995). Algebraic Riccati equations, Clarendon press

[29] G.A. Leonov, D.V. Ponomarenko, V.B. Smirnova (1996). Frequency-domain methods for nonlinear analysis. Theory and applications, World Scientific Publishing Co.

[30] S.-I. Niculescu, J.-M. Dion, L. Dugard, H. Li (1996). Asymptotic stability sets for linear systems with commensurable delays: a matrix pencil approach, IEEE/IMACS CESA'96, Lille, France

[31] D. Peaucelle, D. Arzelier, O. Bachelier, J. Bernussou (2000). A new robust D-stability condition for real convex polytopic uncertainty, Systems and Control Letters 40 no 1, 21-30

[32] D.C.W. Ramos, P.L.D. Peres (2001). An LMI approach to compute robust stability domains for uncertain linear systems, Proc. ACC, Arlington (Virginia), 4073-4078

[33] A. Rantzer (1996). On the Kalman-Yakubovich-Popov lemma, Syst. Contr. Lett. 28 no 1, 7-10

[34] A. Rantzer, M. Johansson (2000). Piecewise linear quadratic optimal control, IEEE Trans. Automat. Control 45 no $4,629-637$

$\mathrm{RR} \mathrm{n}^{\circ} 4316$ 
[35] G. Szegö, R.E. Kalman (1963). Sur la stabilité absolue d'un système d'équations aux différences finies, Comp. Rend. Acad. Sci. 257 no 2, 338-390

[36] M. Sznaier, P.A. Parrilo (1999). On the gap between $\mu$ and its upper bound for systems with repeated uncertainty blocks, Proc. of 38th IEEE CDC, Phoenix (Arizona), 4511-4516

[37] O. Toker, H. Özbay (1996). Complexity issues in robust stability of linear delay-differential systems, Math. Control Signals Systems 9 no 4, 386-400

[38] O. Toker, H. Özbay (1998). On the complexity of purely complex $\mu$ computation and related problems in multidimensional systems, IEEE Trans. Automat. Control 43 no 3, 409-414

[39] S. Treil, The gap between complex structured singular value $\mu$ and its upper bound is infinite, http://www.math.msu.edu/treil/papers/mu/mu-abs.html

[40] A. Trofino (1999). Parameter dependent Lyapunov functions for a class of uncertain linear systems: a LMI approach, Proc. of 38th IEEE CDC, Phoenix (Arizona), 2341-2346

[41] A. Trofino, C.E. de Souza (1999). Bi-quadratic stability of uncertain linear systems, Proc. of 38th IEEE $C D C$, Phoenix (Arizona)

[42] L. Xie, S. Shishkin, M. Fu (1997). Piecewise Lyapunov functions for robust stability of linear timevarying systems, Syst. Contr. Lett. 31 no 3, 165-171

[43] V.A. Yakubovich (1962). Solution of certain matrix inequalities in the stability theory of nonlinear control systems, Dokl. Akad. Nauk. SSSR 143, 1304-1307 (English translation in Soviet Math. Dokl. 3, $620-623(1962))$

[44] J. Zhang, C.R. Knospe, P. Tsiotras (2001). Stability of time-delay systems: equivalence between Lyapunov and scaled small-gain conditions, IEEE Trans. Automat. Control 46 no 3, 482-486

[45] K. Zhou, with J.C. Doyle, K. Glover (1996). Robust and optimal control, Prentice Hall 


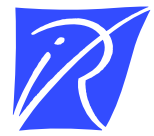

Unité de recherche INRIA Rocquencourt Domaine de Voluceau - Rocquencourt - BP 105 - 78153 Le Chesnay Cedex (France)

Unité de recherche INRIA Lorraine : LORIA, Technopôle de Nancy-Brabois - Campus scientifique 615, rue du Jardin Botanique - BP 101 - 54602 Villers-lès-Nancy Cedex (France)

Unité de recherche INRIA Rennes : IRISA, Campus universitaire de Beaulieu - 35042 Rennes Cedex (France)

Unité de recherche INRIA Rhône-Alpes : 655, avenue de l'Europe - 38330 Montbonnot-St-Martin (France)

Unité de recherche INRIA Sophia Antipolis : 2004, route des Lucioles - BP 93 - 06902 Sophia Antipolis Cedex (France)

INRIA - Domaine de Voluceau - Rocquencourt, BP 105 - 78153 Le Chesnay Cedex (France)

http://www.inria.fr

ISSN 0249-6399 